\title{
Spacelike mean curvature one surfaces in de Sitter 3-space
}

\author{
S. Fujimori, W. Rossman, M. Umehara, K. Yamada and \\ S.-D. YANG
}

The first author studied spacelike constant mean curvature one (CMC-1) surfaces in the de Sitter 3 -space $S_{1}^{3}$ when the surfaces have no singularities except within some compact subsets and are of finite total curvature on the complement of this compact subset. However, there are many CMC- 1 surfaces whose singular sets are not compact. In fact, such examples have already appeared in the construction of trinoids given by Lee and the last author via hypergeometric functions.

In this paper, we improve the Osserman-type inequality given by the first author. Moreover, we shall develop a fundamental framework that allows the singular set to be non-compact, and then will use it to investigate the global behavior of CMC-1 surfaces.

\section{Introduction}

A holomorphic map $F: M^{2} \rightarrow \mathrm{SL}_{2} C$ of a Riemann surface $M^{2}$ into the complex Lie group $\mathrm{SL}_{2} C$ is called null if $\operatorname{det}(d F / d z)$ vanishes identically, where $z$ is a local complex coordinate of $M^{2}$. We consider two projections, one into the hyperbolic 3 -space

$$
\pi_{\mathrm{H}}: \mathrm{SL}_{2} \boldsymbol{C} \longrightarrow H^{3}=\mathrm{SL}_{2} \boldsymbol{C} / \mathrm{SU}_{2}
$$

and the other into the de Sitter 3-space

$$
\pi_{\mathrm{S}}: \mathrm{SL}_{2} \boldsymbol{C} \longrightarrow S_{1}^{3}=\mathrm{SL}_{2} \boldsymbol{C} / \mathrm{SU}_{1,1},
$$

where the definition of $\mathrm{SU}_{1,1}$ is in Appendix $\mathrm{B}$. It is well known that the projection of a holomorphic null immersion into $H^{3}$ by $\pi_{\mathrm{H}}$ gives a conformal constant mean curvature one (CMC-1) immersion (see [3,6,21]). Moreover, conformal CMC-1 immersions are always given locally in such a manner.

On the other hand, spacelike CMC-1 surfaces given by the projection of holomorphic null immersions into $S_{1}^{3}$ by $\pi_{\mathrm{S}}$ can have singularities, and 
are called CMC-1 faces. We work with this class of surfaces that is larger than the class of CMC-1 immersions. In fact, the class of CMC-1 immersions is too small, since there is only one, up to congruency, complete spacelike CMC-1 immersion $[2,17]$, which we call an $S_{1}^{3}$-horosphere. (We also give a simple proof of this here. See Remark 1.15)

The relationship between CMC-1 surfaces in $H^{3}$ and CMC- 1 faces in $S_{1}^{3}$ is analogous to that between minimal surfaces in Euclidean 3-space $\boldsymbol{R}^{3}$ and spacelike maximal surfaces with singularities in the Lorentz-Minkowski 3 -space $\boldsymbol{R}_{1}^{3}$ (called maxfaces [24]). Note that maximal surfaces also admit a Weierstrass-type representation formula ([11]). As in the case of maxfaces (see [24]), the first author [7] investigated the global behavior of CMC-1 faces in $S_{1}^{3}$, in particular proving an Osserman-type inequality for complete CMC-1 faces of finite type whose ends are all elliptic, where a complete end of a CMC-1 face is called elliptic, parabolic or hyperbolic if the monodromy matrix of the holomorphic lift $F: M^{2} \rightarrow \mathrm{SL}_{2} C$ is elliptic, parabolic or hyperbolic, respectively (see Section 1). One of our main results is the following, which implies that the ellipticity or parabolicity of ends follows from completeness:

Theorem 0.1. A complete end of a CMC-1 face in $S_{1}^{3}$ is never hyperbolic, so must be either elliptic or parabolic. Moreover, the total curvature over a neighborhood of such an end is finite.

We remark that there exist incomplete elliptic and parabolic ends.

It is remarkable that just completeness of an end is sufficient to conclude that it has finite total curvature. This is certainly not the case for CMC-1 surfaces in $H^{3}$ nor for minimal surfaces in $\boldsymbol{R}^{3}$, but is similar to the case of maximal surfaces in $\boldsymbol{R}_{1}^{3}$ [24]. Although the asymptotic behavior of regular elliptic CMC-1 ends in $S_{1}^{3}$ is investigated in [7], there do also exist complete parabolic ends, and to describe them, a much deeper analysis is needed, which we will conduct in this article.

As an application of Theorem 0.1, we prove the following Osserman-type inequality, which improves the result of [7] by removing the assumptions of finite type and ellipticity of ends:

Theorem 0.2. Suppose a CMC-1 face $f: M^{2} \rightarrow S_{1}^{3}$ is complete. Then there exist a compact Riemann surface $\bar{M}^{2}$ and a finite number of points $p_{1}, \ldots, p_{n} \in \bar{M}^{2}$ such that $M^{2}$ is biholomorphic to $\bar{M}^{2} \backslash\left\{p_{1}, \ldots, p_{n}\right\}$, and

$$
2 \operatorname{deg}(G) \geq-\chi\left(\bar{M}^{2}\right)+2 n,
$$


where $G$ is the hyperbolic Gauss map of $f$ and $\chi\left(\bar{M}^{2}\right)$ is the Euler characteristic of $\bar{M}^{2}$. Furthermore, equality holds if and only if each end is regular and properly embedded.

CMC-1 trinoids in $S_{1}^{3}$ were constructed by Lee and the last author using hypergeometric functions [15], and those trinoids with elliptic ends are complete in the sense of [7], and attain equality in $(*)$. However, those having other types of ends are not complete, as their singular sets are not compact. For this reason, our goal is not only to prove the above two theorems, but also to extend the framework for CMC-1 surfaces to include a larger class of surfaces, relaxing the immersedness and completeness conditions. If $M^{2}$ is of finite topology, i.e., if $M$ is diffeomorphic to a compact Riemann surface $\bar{M}^{2}$ with finitely many punctures $p_{1}, \ldots, p_{n}$, and if a CMC-1 face $f: M^{2} \rightarrow S_{1}^{3}$ is weakly complete, whose precise definition will be given in Section 1 , we say that $f$ is a weakly complete CMC-1 face of finite topology. We shall develop the framework under this more general notion, which includes all the trinoids in [15].

In Section 1, we recall definitions and basic results. In Section 2, we investigate the monodromy of the hyperbolic metrics on a punctured disk around an end. As an application, we prove Theorem 0.1 in Section 3. In Section 4, we give a geometric interpretation of the hyperbolic Gauss map. In Section 5, we investigate the asymptotic behavior of regular parabolic ends, and prove Theorem 0.2. In Appendix A, we prove meromorphicity of the Hopf differential for complete CMC-1 faces. In Appendix B, we explain the conjugacy classes of $\mathrm{SU}_{1,1}$.

Generic singularities of CMC-1 faces are classified in [9]. A CMC-1 face is called embedded (in the wider sense) if it is embedded outside of some compact set of $S_{1}^{3}$. Examples of complete embedded CMC-1 faces are given in [8] as deformations of maxfaces in the Lorentz-Minkowski 3-space $\boldsymbol{R}_{1}^{3}$.

\section{Preliminaries}

\section{The representation formula}

Let $\boldsymbol{R}_{1}^{4}$ be the Lorentz-Minkowski space of dimension 4, with the Lorentz metric

$$
\left\langle\left(x_{0}, x_{1}, x_{2}, x_{3}\right),\left(y_{0}, y_{1}, y_{2}, y_{3}\right)\right\rangle=-x_{0} y_{0}+x_{1} y_{1}+x_{2} y_{2}+x_{3} y_{3} .
$$


Then the de Sitter 3 -space is

$$
S_{1}^{3}=\left\{\left(x_{0}, x_{1}, x_{2}, x_{3}\right) \in \boldsymbol{R}_{1}^{4} ;-x_{0}^{2}+x_{1}^{2}+x_{2}^{2}+x_{3}^{2}=1\right\}
$$

with metric induced from $\boldsymbol{R}_{1}^{4}$, which is a simply connected Lorentzian 3 -manifold with constant sectional curvature 1 . We identify $\boldsymbol{R}_{1}^{4}$ with the set of $2 \times 2$ Hermitian matrices $\operatorname{Herm}(2)=\left\{X^{*}=X\right\}\left(X^{*}:={ }^{t} \bar{X}\right)$ by

$$
X=\left(x_{0}, x_{1}, x_{2}, x_{3}\right) \leftrightarrow X=\sum_{k=0}^{3} x_{k} e_{k}=\left(\begin{array}{cc}
x_{0}+x_{3} & x_{1}+\mathrm{i} x_{2} \\
x_{1}-\mathrm{i} x_{2} & x_{0}-x_{3}
\end{array}\right),
$$

where

$$
e_{0}=\left(\begin{array}{ll}
1 & 0 \\
0 & 1
\end{array}\right), \quad e_{1}=\left(\begin{array}{ll}
0 & 1 \\
1 & 0
\end{array}\right), \quad e_{2}=\left(\begin{array}{rr}
0 & \mathrm{i} \\
-\mathrm{i} & 0
\end{array}\right), \quad e_{3}=\left(\begin{array}{rr}
1 & 0 \\
0 & -1
\end{array}\right)
$$

and $\mathrm{i}=\sqrt{-1}$. Then $S_{1}^{3}$ is

$$
S_{1}^{3}=\left\{X ; X^{*}=X, \operatorname{det} X=-1\right\}=\left\{A e_{3} A^{*} ; A \in \mathrm{SL}_{2} C\right\}
$$

with the metric

$$
\langle X, Y\rangle=-\frac{1}{2} \operatorname{trace}\left(X e_{2}\left({ }^{t} Y\right) e_{2}\right), \quad\langle X, X\rangle=-\operatorname{det} X .
$$

The projection $\pi_{\mathrm{S}}: \mathrm{SL}_{2} C \rightarrow S_{1}^{3}$ mentioned in the introduction is written explicitly as $\pi_{\mathrm{S}}(A)=A e_{3} A^{*}$. Note that the hyperbolic 3 -space $H^{3}$ is given by $H^{3}=\left\{A A^{*} ; A \in \mathrm{SL}_{2} C\right\}$ and the projection is $\pi_{\mathrm{H}}(A)=A A^{*}$.

An immersion into $S_{1}^{3}$ is called spacelike if the induced metric on the immersed surface is positive definite. The complex Lie group $\mathrm{SL}_{2} C$ acts isometrically on $\operatorname{Herm}(2)=\boldsymbol{R}_{1}^{4}$, as well as $S_{1}^{3}$, by

$$
\operatorname{Herm}(2) \ni X \longmapsto A X A^{*}, \quad A \in \mathrm{SL}_{2} C .
$$

In fact, $\mathrm{PSL}_{2} \boldsymbol{C}=\mathrm{SL}_{2} \boldsymbol{C} /\{ \pm \mathrm{id}\}$ is isomorphic to the identity component $\mathrm{SO}_{3,1}^{+}$of the isometry group $\mathrm{O}_{3,1}$ of $S_{1}^{3}$. Note that each element of $\mathrm{SO}_{3,1}^{+}$ corresponds to an orientation preserving and orthochronous (i.e., time orientation preserving) isometry. The group of orientation preserving isometries of $S_{1}^{3}$ is generated by $\mathrm{PSL}_{2} \boldsymbol{C}$ and the map

$$
S_{1}^{3} \ni X \longmapsto-X \in S_{1}^{3} .
$$

Aiyama-Akutagawa [1] gave a Weierstrass-type representation formula in terms of holomorphic data for spacelike CMC-1 immersions in $S_{1}^{3}$. The 
first author [7] extended the notion of CMC-1 surfaces as follows, like as for the case of maximal surfaces in the Lorentz-Minkowski space [24].

Definition 1.1 [7]. Let $M^{2}$ be a 2-manifold. A $C^{\infty}$-map $f: M^{2} \rightarrow S_{1}^{3}$ is called a $C M C-1$ face if

(1) there exists an open dense subset $W \subset M^{2}$ such that $\left.f\right|_{W}$ is a spacelike CMC-1 immersion.

(2) For any singular point (that is, a point where the induced metric degenerates) $p$, there exists a $C^{1}$-differentiable function $\lambda: U \cap W \rightarrow(0, \infty)$, defined on the intersection of neighborhood $U$ of $p$ with $W$, such that $\lambda d s^{2}$ extends to a $C^{1}$-differentiable Riemannian metric on $U$, where $d s^{2}$ is the first fundamental form, i.e., the pull-back of the metric of $S_{1}^{3}$ by $f$.

(3) $d f(p) \neq 0$ for any $p \in M^{2}$.

Remark 1.1. Although the original definition of CMC-1 faces in [7] assumed the orientability of the source manifold, our definition here does not. However, this difference is not of an essential nature. In fact, for any CMC-1 face $f: M^{2} \rightarrow S_{1}^{3}, M^{2}$ is automatically orientable. (See [12].)

Remark 1.2. A $C^{\infty}$-map $f: M^{2} \rightarrow S_{1}^{3}$ is called a frontal if $f$ lifts to a $C^{\infty}$ map $L_{f}: M^{2} \rightarrow P\left(T^{*} S_{1}^{3}\right)$ such that $d L_{f}\left(T M^{2}\right)$ lies in the canonical contact hyperplane field on $P\left(T^{*} S_{1}^{3}\right)$. Moreover, $f$ is called a wave front or a front if $L_{f}$ is an immersion, that is, $L_{f}\left(M^{2}\right)$ is a Legendrian submanifold. If a frontal $L_{f}$ can lift up to a smooth map into $T^{*} S_{1}^{3}, f$ is called co-orientable, and otherwise it is called non-co-orientable. Wave fronts are a canonical class for investigating flat surfaces in the hyperbolic 3-space $H^{3}$. In fact, like for CMC-1 faces (see Theorem 0.2 in the introduction), an Osserman-type inequality holds for flat fronts in $H^{3}$ (see [14]). Although our CMC-1 faces belong to a special class of horospherical linear Weingarten surfaces (cf. [12]), they may not be (wave) fronts in general, but are co-orientable frontals. In particular, there is a globally defined non-vanishing normal vector field $\nu$ on the whole of $M^{2}$ for a given CMC-1 face $f: M^{2} \rightarrow S_{1}^{3}$. It should be remarked that the limiting tangent plane at each singular point contains a lightlike direction, that is, a CMC-1 face is not spacelike on the singular set.

An oriented 2-manifold $M^{2}$ on which a CMC-1 face $f: M^{2} \rightarrow S_{1}^{3}$ is defined always has a complex structure (see [7]). Since CMC-1 faces are all orientable and co-orientable (cf. [12]), from now on, we will treat $M^{2}$ as 
a Riemann surface, and we can assume the existence of a globally defined non-vanishing normal vector field. The representation formula in [1] can be extended for CMC-1 faces as follows:

Theorem 1.1 ([7], Theorem 1.9). Let $\widetilde{M}^{2}$ be a simply connected Riemann surface. Let $g$ be a meromorphic function and $\omega$ a holomorphic 1-form on $\widetilde{M^{2}}$ such that

$$
d \hat{s}^{2}=\left(1+|g|^{2}\right)^{2}|\omega|^{2}
$$

is a Riemannian metric on $\widetilde{M}^{2}$ and $|g|$ is not identically 1. Take a holomorphic immersion $F=\left(F_{j k}\right): \widetilde{M}^{2} \rightarrow \mathrm{SL}_{2} C$ satisfying

$$
F^{-1} d F=\left(\begin{array}{cc}
g & -g^{2} \\
1 & -g
\end{array}\right) \omega .
$$

Then $f: \widetilde{M^{2}} \rightarrow S_{1}^{3}$ defined by

$$
f=\pi_{\mathrm{S}} \circ F:=F e_{3} F^{*}
$$

is a CMC-1 face. The induced metric $d s^{2}$ on $\widetilde{M}^{2}$, the second fundamental form II, and the Hopf differential $Q$ of $f$ are given as follows:

$$
d s^{2}=\left(1-|g|^{2}\right)^{2}|\omega|^{2}, \quad I I=Q+\bar{Q}+d s^{2}, \quad Q=\omega d g .
$$

The singularities of the CMC-1 face occur at points where $|g|=1$.

Conversely, for any $C M C-1$ face $f: \widetilde{M}^{2} \rightarrow S_{1}^{3}$, there exist a meromorphic function $g$ (with $|g|$ not identically 1) and a holomorphic 1-form $\omega$ on $\widetilde{M^{2}}$ so that $d \hat{s}^{2}$ is a Riemannian metric on $\widetilde{M}^{2}$ and (1.7) holds, where $F: \widetilde{M}^{2} \rightarrow \mathrm{SL}_{2} \boldsymbol{C}$ is an immersion satisfying (1.6).

Remark 1.3. By definition, CMC-1 faces have dense regular sets. However, the projection of null holomorphic immersions might not have dense regular sets, in general. Such an example has been given in ([7], Remark 1.8). Fortunately, we can explicitly classify such degenerate examples, as follows: Let $M^{2}$ be a connected Riemann surface and $F: M^{2} \rightarrow \mathrm{SL}_{2} C$ be a null immersion. We assume that the set of singular points of the corresponding map

$$
f=\mathrm{Fe}_{3} \mathrm{~F}^{*}: M^{2} \longrightarrow S_{1}^{3}
$$

has an interior point. Then the secondary Gauss map $g$ is constant on $M^{2}$ and $|g|=1$. Without loss of generality, we may assume $g=1$. Since $F$ is 
an immersion, $\left(1+|g|^{2}\right)^{2}|\omega|^{2}$ is positive definite. Then $\omega \neq 0$ everywhere. Hence for each $p \in M^{2}$, one can take a complex coordinate $z$ such that $\omega=d z$ near $p$. Then $F$ is a solution of

$$
F^{-1} d F=\left(\begin{array}{cc}
1 & -1 \\
1 & -1
\end{array}\right) d z
$$

Without loss of generality, we may assume that $F(0)=\left(\begin{array}{cc}1 / 2 & 1 / 2 \\ -1 & 1\end{array}\right)$. Then we have

$$
F=\left(\begin{array}{cc}
z+1 / 2 & -z+1 / 2 \\
-1 & 1
\end{array}\right)
$$

and the corresponding map $f$ is computed as

$$
f=F e_{3} F^{*}=\left(\begin{array}{cc}
2 \operatorname{Re} z & -1 \\
-1 & 0
\end{array}\right)
$$

whose image is a lightlike line in $S_{1}^{3}$. Thus, we have shown that the image of any degenerate CMC-1 surface is a part of a lightlike line.

Remark 1.4. Theorem 1.1 is an analogue of the Bryant representation for CMC-1 surfaces in $H^{3}$, which explains why CMC-1 surfaces in both $H^{3}$ and $S_{1}^{3}$ are characterized by the projections $\pi_{\mathrm{H}} \circ F$ and $\pi_{\mathrm{S}} \circ F$. The CMC-1 surfaces in $H^{3}$ and $S_{1}^{3}$ are both typical examples in the class of linear Weingarten surfaces. A Bryant-type representation formula for linear Weingarten surfaces was recently given by Gálvez et al. [10].

Remark 1.5. Following the terminology of [21], $g$ is called a secondary Gauss map of $f$. The pair $(g, \omega)$ is called Weierstrass data of $f$, and $F$ is called a holomorphic null lift of $f$.

The holomorphic 2-differential $Q$ as in (1.8) is called the Hopf differential of $f$. In analogy with the theory of CMC-1 surfaces in $H^{3}$, the meromorphic function

$$
G:=\frac{d F_{11}}{d F_{21}}=\frac{d F_{12}}{d F_{22}}
$$

is called the hyperbolic Gauss map. A geometric meaning for the hyperbolic Gauss map is given in Section 4. 
Remark 1.6. Corresponding to Theorem 1.1, a Weierstrass-type representation formula is known for spacelike maximal surfaces in $\boldsymbol{R}_{1}^{3}$ [11]. In fact, the Weierstrass data $(g, \omega)$ as in Theorem 1.1 defines null curves in $\boldsymbol{C}^{3}$ by

$$
F_{0}(z):=\int_{z_{0}}^{z}\left(-2 g, 1+g^{2}, \mathrm{i}\left(1-g^{2}\right)\right) \omega .
$$

Any maxface (see [24] for the definition) is locally obtained as the real part of some $F_{0}$. Moreover, their first fundamental forms and Hopf differentials are given by (1.8). The meromorphic function $g$ can be identified with the Lorentzian Gauss map.

Remark 1.7. Let $G, g$ be meromorphic functions on a Riemann surface. Set

$$
F=\left(\begin{array}{cc}
G \frac{d a}{d G}-a & G \frac{d b}{d G}-b \\
\frac{d a}{d G} & \frac{d b}{d G}
\end{array}\right), \quad a=\sqrt{\frac{d G}{d g}}, \quad b=-g a .
$$

Then $F$ is a meromorphic null map with hyperbolic and secondary Gauss maps $G$ and $g$ respectively. Formula (1.10) is called Small's formula $[13,19]$.

Remark 1.8. The holomorphic null lift $F$ of a CMC-1 face $f$ is unique up to right-multiplication by matrices in $\mathrm{SU}_{1,1}$, that is, for each $A \in \mathrm{SU}_{1,1}$, the projection of $F A^{-1}$ is also $f$. Under the transformation $F \mapsto F A^{-1}$, the secondary Gauss map $g$ changes by a Möbius transformation:

$$
g \longmapsto A \star g:=\frac{A_{11} g+A_{12}}{A_{21} g+A_{22}}, \quad A=\left(\begin{array}{ll}
A_{11} & A_{12} \\
A_{21} & A_{22}
\end{array}\right) .
$$

The conditions $|g|=1,|g|>1,|g|<1$ are invariant under this transformation.

In particular, let $f: M^{2} \rightarrow S_{1}^{3}$ be a CMC-1 face of a (not necessarily simply connected) Riemann surface $M^{2}$. Then the holomorphic null lift $F$ is defined only on the universal cover $\widetilde{M}^{2}$ of $M^{2}$. Take a deck transformation $\tau \in \pi_{1}\left(M^{2}\right)$ in $\widetilde{M}^{2}$. Since $\pi_{\mathrm{S}} \circ F=\pi_{\mathrm{S}} \circ F \circ \tau$, there exists a $\tilde{\rho}(\tau) \in \mathrm{SU}_{1,1}$ such that

$$
F \circ \tau=F \tilde{\rho}(\tau) .
$$

The induced homomorphism $\tilde{\rho}: \pi_{1}\left(M^{2}\right) \rightarrow \mathrm{SU}_{1,1}$ is called the monodromy representation, which induces a $\mathrm{PSU}_{1,1}$-representation

$$
\rho: \pi_{1}\left(M^{2}\right) \rightarrow \mathrm{PSU}_{1,1}=\mathrm{SU}_{1,1} /\{ \pm 1\}
$$


satisfying

$$
g \circ \tau^{-1}=\rho(\tau) \star g
$$

Remark 1.9. The action $F \mapsto B F, B \in \mathrm{SL}_{2} \boldsymbol{C}$, induces a rigid motion $f \mapsto$ $B f B^{*}$ in $S_{1}^{3}$, and the isometric motion $f \mapsto-f$ as in (1.4) corresponds to

$$
F \longmapsto F^{\natural}=F\left(\begin{array}{ll}
0 & \mathrm{i} \\
\mathrm{i} & 0
\end{array}\right)=\mathrm{i} F e_{1}
$$

The secondary Gauss map of $F^{\natural}$ is $1 / g$.

Remark 1.10. Let $K_{d s^{2}}$ be the Gaussian curvature of $d s^{2}$ on the set of regular points of $f$. Then

$$
d \sigma^{2}:=K_{d s^{2}} d s^{2}=\frac{4|d g|^{2}}{\left(1-|g|^{2}\right)^{2}}
$$

is a pseudometric of constant curvature -1 , which degenerates at isolated umbilic points. We have

$$
d \sigma^{2} \cdot d s^{2}=4|Q|^{2}
$$

Remark 1.11. The metric

$$
d s_{\#}^{2}:=\operatorname{trace}\left(\alpha_{\#}\right)\left(\alpha_{\#}\right)^{*}=\left(1+|G|^{2}\right)^{2}\left|\frac{Q}{d G}\right|^{2} \quad\left(\alpha_{\#}:=\left(F^{-1}\right)^{-1} d\left(F^{-1}\right)\right)
$$

is induced from the canonical Hermitian metric of $\mathrm{SL}_{2} C$ via $F^{-1}: \widetilde{M}^{2} \rightarrow$ $\mathrm{SL}_{2} C$. When the CMC-1 face is defined on $M^{2}, G$ and $Q$ are as well, so $d s_{\#}^{2}$ is well defined on $M^{2}$, and is called the lift metric. It is nothing but the dual metric of the CMC-1 surface $\pi_{\mathrm{H}} \circ F$ in $H^{3}$, see [23].

\section{Completeness}

We now define two different notions of completeness for CMC-1 faces as follows:

Definition 1.2. We say a CMC-1 face $f: M^{2} \rightarrow S_{1}^{3}$ is complete if there exists a symmetric 2 -tensor field $T$ which vanishes outside a compact subset $C \subset M^{2}$ such that the sum $T+d s^{2}$ is a complete Riemannian metric on $M^{2}$. 
See [7], with similar definitions in [14] for flat fronts in $H^{3}$ and in [24] for maxfaces.

Definition 1.3. We say that $f$ is weakly complete if it is congruent to an $S_{1}^{3}$-horosphere or if the lift metric (1.17) is a complete Riemannian metric on $M^{2}$.

Here, the $S_{1}^{3}$-horosphere is the totally umbilic CMC-1 surface, which is also the only complete CMC-1 immersed surface (see Remark 1.15). It has the Weierstrass data $g=c=$ constant $(|c| \neq 1)$ and $\omega=d z$. The metric $d s_{\#}^{2}$ of an $S_{1}^{3}$-horosphere cannot be defined by (1.17) as $G$ is constant and $Q$ is identically 0 .

Definition 1.4. We say that $f$ is of finite type if there exists a compact set $C$ of $M^{2}$ such that the first fundamental form $d s^{2}$ is positive definite and has finite total (absolute) curvature on $M^{2} \backslash C$.

Let $f: M^{2} \rightarrow S_{1}^{3}$ be a CMC-1 face of finite topology, that is, $M^{2}$ is diffeomorphic to a compact Riemann surface $\bar{M}^{2}$ with a finite number of points $\left\{p_{1}, \ldots, p_{n}\right\} \subset \bar{M}^{2}$ excluded. We can take a punctured neighborhood $\Delta_{j}^{*}$ of $p_{j}$ which is biholomorphic to either the punctured unit disk $\Delta^{*}=\{z \in$ $C ; 0<|z|<1\}$ or an annular domain, and $p_{j}$ is called a puncture-type end or an annular end, respectively.

Proposition 1.1. Let $f: M^{2} \rightarrow S_{1}^{3}$ be a $C M C$-1 face. If $f$ is complete, then

(1) the singular set of $f$ is compact,

(2) $f$ is weakly complete,

(3) $M^{2}$ has finite topology and each end is of puncture-type.

Proof. (1) is obvious. If $f$ is totally umbilic, it is congruent to an $S_{1}^{3}$ horosphere and the assertion is obvious. So we assume the Hopf differential $Q$ does not vanish identically. Since the Gaussian curvature of $f$ is nonnegative, completeness implies (3) by the appendix of [24]. So we shall now prove that completeness implies weak completeness: fix an end $p_{j}$ of $f$. By an appropriate choice of a coordinate $z$, the restriction of $f$ to a neighborhood of $p_{j}$ is $f_{j}: \Delta^{*} \rightarrow S_{1}^{3}$. We denote by $d \hat{s}^{2}$ the induced metric of the corresponding CMC-1 surface $\hat{f}_{j}=F F^{*}: \widetilde{\Delta}^{*} \rightarrow H^{3}$ into hyperbolic 3 -space (see $(1.5)$, where $\widetilde{\Delta}^{*}$ is the universal cover of $\left.\Delta^{*}\right)$. Take a path $\gamma:[0,1) \rightarrow \Delta^{*}$ such that $\gamma(t) \rightarrow 0$ as $t \rightarrow 1$. Then by (1.5) and (1.8), $d \hat{s}^{2} \geq d s^{2}$ holds, and 
hence completeness of $f$ implies that each lift $\tilde{\gamma}:[0,1) \rightarrow \widetilde{\Delta}^{*}$ of $\gamma$ has infinite length with respect to $d \hat{s}^{2}$. Here, $d \hat{s}^{2}$ and $d s_{\#}^{2}=\left(1+|G|^{2}\right)^{2}|Q / d G|^{2}$ are the pull-backs of the canonical Hermitian metric of $\mathrm{SL}_{2} C$ by $F$ and $F^{-1}$, respectively. $\mathrm{Yu}[26]$ showed that completeness of these two metrics are equivalent. Hence, $\tilde{\gamma}$ has infinite length with respect to the metric $d s_{\#}^{2}$. Since $d s_{\#}^{2}$ is well defined on $\Delta^{*}, \gamma$ also has infinite length with respect to $d s_{\#}^{2}$, that is, the metric $d s_{\#}^{2}$ on $\Delta^{*}$ is complete at 0 . Thus, $f_{j}$ is a weakly complete end.

For further properties of complete ends, see Theorems 3.1 and 3.2.

Remark 1.12. The converse of Proposition 1.1 is true (see [25]). Our definition of weak completeness of CMC-1 faces is somewhat more technical than that of maxfaces [24], but it is the correctly corresponding concept in $S_{1}^{3}$ : for data $(g, \omega)$, weak completeness of the associated maxface in $\boldsymbol{R}_{1}^{3}$ is equivalent to that of the CMC-1 face in $S_{1}^{3}$.

Remark 1.13. The CMC-1 trinoids in $S_{1}^{3}$ constructed in [15] are all weakly complete (sometimes complete as well) and all ends are $g$-regular, see Section 3.

Remark 1.14. The Hopf differential $Q$ of a complete CMC-1 face $f$ : $M^{2} \rightarrow S_{1}^{3}$ is meromorphic on its compactification $\bar{M}^{2}$, even without assuming that all ends of $f$ are regular. See Appendix A. It should be remarked that for CMC-1 surfaces in the hyperbolic 3-space, finiteness of total curvature is needed to show the meromorphicity of $Q$ (see [3]).

\section{Monodromy of ends of CMC-1 faces}

For any real number $t$, we set

$$
\begin{aligned}
\Lambda_{\mathrm{e}}(t) & :=\left(\begin{array}{cc}
e^{\mathrm{i} t} & 0 \\
0 & e^{-\mathrm{i} t}
\end{array}\right) \\
\Lambda_{\mathrm{p}}(t) & :=\left(\begin{array}{cc}
1+\mathrm{i} t & -\mathrm{i} t \\
\mathrm{i} t & 1-\mathrm{i} t
\end{array}\right), \\
\Lambda_{\mathrm{h}}(t) & :=\left(\begin{array}{cc}
\cosh t & \sinh t \\
\sinh t & \cosh t
\end{array}\right) .
\end{aligned}
$$

A matrix in $\mathrm{SU}_{1,1}$ is called

(1) elliptic if it is conjugate to $\Lambda_{\mathrm{e}}(t)(t \in(-\pi, \pi])$ in $\mathrm{SU}_{1,1}$,

(2) parabolic if it is conjugate to $\pm \Lambda_{\mathrm{p}}(t)(t \in \boldsymbol{R} \backslash\{0\})$ in $\mathrm{SU}_{1,1}$, and

(3) hyperbolic if it is conjugate to $\pm \Lambda_{\mathrm{h}}(t)(t>0)$ in $\mathrm{SU}_{1,1}$. 
Any matrix in $\mathrm{SU}_{1,1}$ is of one of these three types, see Appendix B. Note that the parabolic matrices $\Lambda_{\mathrm{p}}\left(t_{1}\right)$ and $\Lambda_{\mathrm{p}}\left(t_{2}\right)$ are conjugate in $\mathrm{SU}_{1,1}$ if and only if $t_{1} t_{2}>0$. Although the set of conjugate classes of parabolic matrices is fully represented by $\left\{ \pm \Lambda_{\mathrm{p}}( \pm 1)\right\}$, we may use various values of $t$ in this paper for the sake of simplicity.

Let $f: M^{2} \rightarrow S_{1}^{3}$ be a weakly complete CMC-1 face of finite topology, where $M^{2}$ is diffeomorphic to a compact Riemann surface $\bar{M}^{2}$ with finitely many punctures $\left\{p_{1}, \ldots, p_{n}\right\}$. Any puncture $p_{j}$, or occasionally a small neighborhood $U_{j}$ of $p_{j}$, is called an end of $f$.

An end is called elliptic, parabolic or hyperbolic when the monodromy matrix $\tilde{\rho}(\tau) \in \mathrm{SU}_{1,1}$ is elliptic, parabolic or hyperbolic, respectively, where $\tilde{\rho}$ is as in Remark 1.8 and $\tau \in \pi_{1}\left(M^{2}\right)$ is the deck transformation corresponding to the counterclockwise loop about $p_{j}$.

\section{The Schwarzian derivative}

Let $(U, z)$ be a local complex coordinate of a Riemann surface $M^{2}$, and $h(z)$ a meromorphic function on $U$. Then

$$
S_{z}(h):=\left(\frac{h^{\prime \prime}}{h^{\prime}}\right)^{\prime}-\frac{1}{2}\left(\frac{h^{\prime \prime}}{h^{\prime}}\right)^{2} \quad\left({ }^{\prime}=\frac{d}{d z}\right)
$$

is the Schwarzian derivative of $h$ with respect to the coordinate $z$.

If $h(z)=a+b(z-p)^{m}+o\left((z-p)^{m}\right)$ at $z=p \in U \quad(b \neq 0)$, where $o\left((z-p)^{m}\right)$ denotes higher order terms, then the positive integer $m$ is called the (ramification) order of $h(z)$, and we have

$$
S_{z}(h)=\frac{1}{(z-p)^{2}}\left(\frac{1-m^{2}}{2}+o(1)\right)
$$

We write $S(h)=S_{z}(h) d z^{2}$, which we also call the Schwarzian derivative. The Schwarzian derivative depends on the choice of local coordinates, but the difference does not, that is, $S\left(h_{1}\right)-S\left(h_{2}\right)$ is a well-defined holomorphic 2-differential.

The Schwarzian derivative is invariant under Möbius transformations: $S(h)=S(A \star h)$ holds for $A \in \mathrm{SL}_{2} C$, where $\star$ denotes the Möbius transformation as in (1.11). Conversely, if $S_{z}(h)=S_{z}(g)$, there exists an $A \in \mathrm{SL}_{2} C$ such that $g=A \star h$. 
Let $f: M^{2} \rightarrow S_{1}^{3}$ be a CMC-1 face with the hyperbolic Gauss map $G$, a secondary Gauss map $g$ and the Hopf differential $Q$. Then

$$
S(g)-S(G)=2 Q
$$

Remark 1.15. Here we give a proof that the only complete CMC-1 immersion is the totally umbilic one, that is, the $S_{1}^{3}$-horosphere, which is simpler than the original proofs in $[2,17]$. (The proof is essentially the same as for the case of maximal surfaces in $\boldsymbol{R}_{1}^{3}$ given in [24, Remark 1.2].) Let $f: M^{2} \rightarrow S_{1}^{3}$ be a complete CMC-1 immersion. Without loss of generality, we may assume that $M^{2}$ is both connected and simply connected. Then the Weierstrass data $(g, \omega)$ as in Theorem 1.1 is single valued on $M^{2}$. Since $f$ has no singular points, we may assume that $|g|<1$ holds on $M^{2}$. Since $\left(1-|g|^{2}\right)^{2}|\omega|^{2}<|\omega|^{2}$, the metric $|\omega|^{2}$ is a complete flat metric on $M^{2}$. Then the uniformization theorem yields that $M^{2}$ is bi-holomorphic to $\boldsymbol{C}$, and $g$ must be a constant function, which implies that the image of $f$ must be totally umbilic.

\section{Monodromy of punctured hyperbolic metrics}

By Remark 1.8, the monodromy of a holomorphic null immersion $F$ is elliptic, parabolic or hyperbolic if and only if the monodromy of its secondary Gauss map $g$ is elliptic, parabolic or hyperbolic, respectively. In this section, in an abstract setting, we give results needed for investigating the behavior of $g$ at a puncture-type end, in terms of the monodromy of $g$.

\section{Lifts of $\mathrm{PSU}_{1,1}$-projective connections on a punctured disk}

Let

$$
\Delta^{*}=\Delta \backslash\{0\}, \quad \text { where } \Delta:=\{z \in C ;|z|<1\},
$$

be the punctured unit disk and $P=p(z) d z^{2}$ a holomorphic 2-differential on $\Delta^{*}$. Then there exists a holomorphic developing map $g_{P}: \widetilde{\Delta}^{*} \rightarrow \boldsymbol{C} \cup\{\infty\}$ such that $S\left(g_{P}\right)=P$, where $\widetilde{\Delta}^{*}$ is the universal cover of $\Delta^{*}$. For any other holomorphic function $h$ such that $S(h)=P$, there exists an $A \in \mathrm{SL}_{2} C$ so that $A \star g_{P}=h$. Thus there exists a matrix $T \in \mathrm{PSL}_{2} C$ such that

$$
g_{P} \circ \tau^{-1}=T \star g_{P}
$$

where $\tau$ is the generator of $\pi_{1}\left(\Delta^{*}\right)$ corresponding to a counterclockwise loop about the origin. We call $T$ the monodromy matrix of $g_{P}$. If there exists a $g_{P}$ so that $T \in \mathrm{PSU}_{1,1}, P$ is called a $\mathrm{PSU}_{1,1}$-projective connection on $\Delta^{*}$ and 
$g_{P}$ is called a $\mathrm{PSU}_{1,1}$-lift of $P$. A PSU $\mathrm{PS}_{1,1}$-projective connection on $\Delta^{*}$ has a removable singularity, a pole or an essential singularity at 0 , and is said to have a regular singularity at 0 if it has at most a pole of order 2 at 0 . (The general definition of projective connections is given in [20,22]. There exist holomorphic 2-differentials on $\Delta^{*}$ which are not $\mathrm{PSU}_{1,1}$-projective connections.) When $T \in \mathrm{PSU}_{1,1}$, it is conjugate to one of the matrices in (1.18). The $\mathrm{PSU}_{1,1}$-projective connection $P$ is then called elliptic, parabolic or hyperbolic when $T$ is elliptic, parabolic or hyperbolic, respectively. This terminology is independent of the choice of $g_{P}$.

By property (1.13), the Schwarzian derivative $S(g)$ of the secondary Gauss map $g$ of a CMC- 1 face is an example of a PSU $\mathrm{PS}_{1,1}$-projective connection.

Note that a PSU 1,1 -lift $g_{P}$ has the PSU $\mathrm{PS}_{1,1}$ ambiguity $g_{P} \mapsto A \star g_{P}$ for $A \in \mathrm{PSU}_{1,1}$. The property that $\left|g_{P}\right|>1$ (resp. $\left|g_{P}\right|<1$ ) is independent of this ambiguity.

Remark 2.1. Let $g_{P}$ be a PSU $\mathrm{PS}_{1,1}$-lift of a $\mathrm{PSU}_{1,1}$-projective connection $P$. Then

$$
\frac{1}{g_{P}}=D \star g_{P} \quad\left(D:=\left(\begin{array}{ll}
0 & \mathrm{i} \\
\mathrm{i} & 0
\end{array}\right)\right)
$$

is also a $\mathrm{PSU}_{1,1}$-lift of $P$, because $D A D^{-1} \in \mathrm{PSU}_{1,1}$ for any $A \in \mathrm{PSU}_{1,1}$. However, $D \notin \mathrm{PSU}_{1,1}$, and one can show that there is no matrix $B \in \mathrm{PSU}_{1,1}$ such that $1 / g_{P}=B \star g_{P}$, that is, $1 / g_{P}$ is not $\mathrm{PSU}_{1,1}$-equivalent to $g_{P}$.

In the rest of this article, as well as in the following proposition, we use

$$
R:=\frac{1}{2}\left(\begin{array}{rr}
1 & 1 \\
\mathrm{i} & -\mathrm{i}
\end{array}\right),
$$

which is motivated by an isomorphism between $\mathrm{SL}_{2} \boldsymbol{R}$ and $\mathrm{SU}_{1,1}$. See Appendix B.

Proposition 2.1. Let $P$ be a $\mathrm{PSU}_{1,1}$-projective connection on $\Delta^{*}$. Then the following assertions hold:

(1) Suppose that $P$ is elliptic. Then,

(i) there exist a real number $\mu$ and a single-valued meromorphic function $h(z)$ on $\Delta^{*}$ such that

$$
g(z):=z^{\mu} h(z)
$$

is a $\mathrm{PSU}_{1,1}$-lift of $P$. 
(ii) $P$ has a regular singularity at $z=0$ if and only if $h(z)$ has at most a pole at $z=0$.

(2) Suppose that $P$ is parabolic and take an arbitrary positive number $t$. Then,

(i) for each $\varepsilon \in\{-1,1\}$, there exists a single-valued meromorphic function $h(z)$ on $\Delta^{*}$ such that

$$
g(z):=R^{-1} \star\left(h(z)-\frac{\varepsilon t}{\pi \mathrm{i}} \log z\right)
$$

is a $\mathrm{PSU}_{1,1}$-lift of $P$.

(ii) The function $h(z)$ has at most a pole at $z=0$ if and only if $P$ has a pole of order exactly 2 at $z=0$.

(iii) $h(z)$ is holomorphic at $z=0$ if and only if $P-d z^{2} /\left(2 z^{2}\right)$ has at most a pole of order 1 at $z=0$.

(iv) When $h(z)$ is holomorphic at $z=0,|g(z)|>1(\operatorname{resp} .|g(z)|<1)$ holds for sufficiently small $|z|$ if and only if $\varepsilon=+1$ (resp. $\varepsilon=-1)$.

(3) Suppose that $P$ is hyperbolic. Then,

(i) there exist a positive number $\mu$ and a single-valued meromorphic function $h(z)$ on $\Delta^{*}$ such that

$$
g(z):=R^{-1} \star\left(z^{\mathrm{i} \mu} h(z)\right)
$$

is a $\mathrm{PSU}_{1,1}$-lift of $P$.

(ii) $h(z)$ has at most a pole at $z=0$ if and only if $P$ has a pole of order exactly 2 at $z=0$.

Remark 2.2. In the statements of Proposition 2.1, the function $z^{\mu}(\mu \in \boldsymbol{C})$ is defined by

$$
z^{\mu}:=\exp (\mu \log z)
$$

where $\log z$ is considered as a function defined on the universal cover $\widetilde{\Delta}^{*}$ of $\Delta^{*}$.

To prove this, we consider the following ordinary differential equation

$$
X^{\prime \prime}+\frac{1}{2} p(z) X=0 \quad\left({ }^{\prime}=\frac{d}{d z}, P=p(z) d z^{2}\right)
$$


If we assume $P(z)$ has a regular singularity at $z=0$, then $p(z)=\alpha z^{-2}(1+$ $o(1))$ for some $\alpha \in \boldsymbol{C}$ and (2.3) has the fundamental system of solutions

$$
\begin{aligned}
& X_{1}(z)=z^{\mu_{1}} \xi_{1}(z) \\
& X_{2}(z)=z^{\mu_{2}} \xi_{2}(z)+k \log z X_{1} \quad\left(\operatorname{Re} \mu_{1} \geq \operatorname{Re} \mu_{2}\right),
\end{aligned}
$$

where $\xi_{j}(z)(j=1,2)$ are holomorphic functions on $\Delta=\{|z|<1\}$ such that $\xi_{j}(0) \neq 0(j=1,2)$. The constant $k \in \boldsymbol{C}$ is called the log-term coefficient and $\mu_{1}, \mu_{2}$ are the solutions of the indicial equation

$$
t(t-1)+\frac{\alpha}{2}=0
$$

If $\mu_{1}-\mu_{2} \notin Z$, then $k$ vanishes. (See [5] or the appendix of [18].) The following lemma is easy to show:

Lemma 2.1. In the above setting, $S\left(g_{0}\right)=P$ if $g_{0}:=X_{2} / X_{1}$.

Proof of Proposition 2.1. Take the matrix $T$ as in (2.1).

We first prove the elliptic case. Since $P$ is elliptic, there exist a $t \in \boldsymbol{R}$ and an $A \in \mathrm{SU}_{1,1}$ such that $A T A^{-1}=\Lambda_{\mathrm{e}}(t)$. So $(A \star g) \circ \tau^{-1}=\mathrm{e}^{2 \mathrm{i} t}(A \star g)$, and $h(z):=z^{t / \pi}(A \star g(z))$ is single-valued on $\Delta^{*}$, proving the first part of (1). If the origin 0 is at most a pole of $h$, a direct calculation shows that $P$ has a regular singularity. To show the converse, we set $g_{0}:=X_{2} / X_{1}$, with $\left\{X_{1}, X_{2}\right\}$ as in (2.4). Then by Lemma 2.1 , we have $S\left(g_{0}\right)=P$. The monodromy matrix $\pm T_{0}$ of $g_{0}$ is conjugate to

$$
\left\{\begin{array}{lc}
\left(\begin{array}{cc}
\mathrm{e}^{\pi \mathrm{i}\left(\mu_{1}-\mu_{2}\right)} & 0 \\
0 & \mathrm{e}^{\pi \mathrm{i}\left(\mu_{2}-\mu_{1}\right)}
\end{array}\right) & (\text { if } k=0), \\
\left(\begin{array}{cc}
1 & -2 \pi \mathrm{i} k \\
0 & 1
\end{array}\right) & (\text { if } k \neq 0) .
\end{array}\right.
$$

Since $P$ is elliptic, the log-term coefficient $k=0$ and $\mu_{2}-\mu_{1} \in \boldsymbol{R}$. Thus

$$
g_{0}(z)=z^{\mu} \frac{\xi_{2}(z)}{\xi_{1}(z)} \quad\left(\mu:=\mu_{2}-\mu_{1}\right) .
$$

Since $S(A \star g)=S\left(g_{0}\right)$, there exists a $B \in \mathrm{SL}_{2} C$ so that $A \star g=B \star g_{0}$. Then

$$
\Lambda_{\mathrm{e}}(t) \star(A \star g)=(A \star g) \circ \tau^{-1}=B \star\left(g_{0} \circ \tau^{-1}\right)=B \Lambda_{\mathrm{e}}(-\pi \mu) B^{-1} \star(A \star g),
$$


so $\Lambda_{\mathrm{e}}(t)= \pm B \Lambda_{\mathrm{e}}(-\pi \mu) B^{-1}$. If $t \equiv 0(\bmod \pi)$, then $A \star g$ is meromorphic, proving (1). Otherwise,

$$
B=\left(\begin{array}{cc}
c & 0 \\
0 & c^{-1}
\end{array}\right) \quad \text { or } \quad\left(\begin{array}{cc}
0 & c \\
-c^{-1} & 0
\end{array}\right)
$$

for some $c \in \boldsymbol{C} \backslash\{0\}$, and (1) (ii) in the proposition follows from

$$
A \star g(z)=c^{2} z^{\mu} \frac{\xi_{2}(z)}{\xi_{1}(z)} \text { or } \quad-c^{2} z^{-\mu} \frac{\xi_{1}(z)}{\xi_{2}(z)},
$$

respectively.

Next, we assume $P$ is parabolic and take a positive number $t$ and $\varepsilon \in\{-1,1\}$. Then by Theorem B.1 and Remark B.2 in Appendix B, there exists a matrix $A \in \mathrm{SU}_{1,1}$ such that $A T A^{-1}$ is one of $\Lambda_{\mathrm{p}}(\varepsilon t),-\Lambda_{\mathrm{p}}(\varepsilon t)$, $\Lambda_{\mathrm{p}}(-\varepsilon t),-\Lambda_{\mathrm{p}}(-\varepsilon t)$. Note that $\Lambda_{\mathrm{p}}(\varepsilon t)$ and $\Lambda_{\mathrm{p}}(-\varepsilon t)$ are not conjugate in $\mathrm{PSU}_{1,1}$. Replacing $g$ with $1 / g$ if $A T A^{-1}= \pm \Lambda_{\mathrm{p}}(-\varepsilon t)$ (see Remark 2.1), we can choose a $\mathrm{PSU}_{1,1}$-lift $g$ such that

$$
A T A^{-1}= \pm \Lambda_{\mathrm{p}}(\varepsilon t) .
$$

Then, $(A \star g) \circ \tau^{-1}=\Lambda_{\mathrm{p}}(\varepsilon t) \star(A \star g)$. Here the \pm -ambiguity of $A T A^{-1}$ does not affect the $\star$-action. Thus,

$$
((R A) \star g) \circ \tau^{-1}=(R A) \star g+2 \varepsilon t, \quad \text { since } \quad R \Lambda_{\mathrm{p}}(\varepsilon t)=\left(\begin{array}{cc}
1 & 2 \varepsilon t \\
0 & 1
\end{array}\right) R .
$$

Hence $h(z):=(R A) \star g+(\varepsilon t /(\pi \mathrm{i})) \log z$ is a single-valued meromorphic function on $\Delta^{*}$, proving the first part of (2) in the proposition. If $h(z)$ has at most a pole at $z=0$, then a direct computation shows that $P$ has a pole of order exactly 2 . Therefore, it suffices to show that $h(z)$ has at most a pole at $z=0$ when $P$ has a regular singularity. We now show this:

We set $g_{0}:=X_{2} / X_{1}$. Since $P$ is parabolic, (2.6) yields that the log-term coefficient $k \neq 0$ and $\mu:=\mu_{2}-\mu_{1} \in Z$ is non-positive. Hence

$$
g_{0}=z^{\mu} \frac{\xi_{2}(z)}{\xi_{1}(z)}+k \log z .
$$

Here $z^{\mu} \xi_{2}(z) / \xi_{1}(z)$ is single-valued on $\Delta^{*}$ and has at most a pole at $z=0$. Take a matrix $B \in \mathrm{SL}_{2} C$ such that $(R A) \star g=B \star g_{0}$. Then

$$
\begin{aligned}
\left(\begin{array}{cc}
1 & 2 \varepsilon t \\
0 & 1
\end{array}\right) \star(R A \star g) & =(R A \star g) \circ \tau^{-1}=B \star\left(g_{0} \circ \tau^{-1}\right) \\
& =B\left(\begin{array}{cc}
1 & -2 \pi \mathrm{i} k \\
0 & 1
\end{array}\right) \star g_{0}=B\left(\begin{array}{cc}
1 & -2 \pi \mathrm{i} k \\
0 & 1
\end{array}\right) B^{-1} \star(R A \star g) .
\end{aligned}
$$


Replacing $X_{2}$ by (i $\left.\varepsilon t /(\pi k)\right) X_{2}$ and renaming (i $\left.\varepsilon t /(\pi k)\right) \xi_{2}$ to $\xi_{2},-2 \pi \mathrm{i} k$ becomes $2 \varepsilon t$, and we have

$$
\left(\begin{array}{cc}
1 & 2 \varepsilon t \\
0 & 1
\end{array}\right)=B\left(\begin{array}{cc}
1 & 2 \varepsilon t \\
0 & 1
\end{array}\right) B^{-1}
$$

and there is no \pm -ambiguity in the above equation, as the eigenvalues of the left-hand matrix must have the same sign as those of the right-hand matrix. Thus we can choose

$$
B=\left(\begin{array}{ll}
1 & c \\
0 & 1
\end{array}\right)
$$

for some $c \in \boldsymbol{C}$, which proves the second part of (2) in the Proposition 2.1. It is easy to see that $h(z)$ is holomorphic at $z=0$ if and only if $\mu_{2}=\mu_{1}$, that is, $\alpha$ in (2.5) is $1 / 2$, which proves the third part of (2). Assume $h$ is holomorphic on $\Delta$. Since the Möbius transformation $z \mapsto R \star z$ maps the disk $\Delta$ onto the upper-half plane $\{z ; \operatorname{Im} z>0\}$, the condition $|g|>1$ (equivalently $|A \star g|>1)$ is equivalent to $\operatorname{Im}(R A \star g)<0$ for all $A \in \mathrm{SU}_{1,1}$. And since $|h|$ is bounded, this is equivalent to $\varepsilon>0$. Thus we obtain the last part of (2).

Next, we assume $P$ is hyperbolic. By Theorem B.1 in Appendix B, there are a matrix $A \in \mathrm{SU}_{1,1}$ and $t>0$ such that $A T A^{-1}=\Lambda_{\mathrm{h}}(t)$ or $-\Lambda_{\mathrm{h}}(t)$. Then $(A \star g) \circ \tau^{-1}=\Lambda_{\mathrm{h}}(t) \star(A \star g)$, which implies $((R A) \star g) \circ \tau^{-1}=\mathrm{e}^{2 t}(R A) \star$ $g$. So $h(z):=z^{-\mathrm{i} t / \pi}(R A) \star g$ is a single-valued meromorphic function in $\Delta^{*}$. This proves the first part of (3). To prove the second part of (3), analogous to the parabolic case, we only need to prove one direction. Suppose that $P$ has a regular singularity. We set $g_{0}:=X_{2} / X_{1}$. Since $P$ is hyperbolic, (2.6) yields that $k=0$ and $\mu_{2}-\mu_{1} \in \mathrm{i} \boldsymbol{R}$. Thus

$$
g_{0}(z)=z^{\mathrm{i} \mu} \frac{\xi_{2}(z)}{\xi_{1}(z)}, \quad \mu:=\mathrm{i}\left(\mu_{1}-\mu_{2}\right) .
$$

Exchanging $X_{1}$ and $X_{2}$ if necessary, we may assume $\mu>0$ without loss of generality. Take a $B \in \mathrm{SL}_{2} C$ such that $R A \star g=B \star g_{0}$. Then we have

$$
\left(\begin{array}{cc}
\mathrm{e}^{t} & 0 \\
0 & \mathrm{e}^{-t}
\end{array}\right) \star(R A \star g)=B \star\left(g_{0} \circ \tau^{-1}\right)=B\left(\begin{array}{cc}
\mathrm{e}^{\pi \mu} & 0 \\
0 & \mathrm{e}^{-\pi \mu}
\end{array}\right) B^{-1} \star(R A \star g),
$$

so

$$
\left(\begin{array}{cc}
\mathrm{e}^{t} & 0 \\
0 & \mathrm{e}^{-t}
\end{array}\right)=B\left(\begin{array}{cc}
\mathrm{e}^{\pi \mu} & 0 \\
0 & \mathrm{e}^{-\pi \mu}
\end{array}\right) B^{-1}
$$


that is, $t= \pm \pi \mu$. As we have assumed that $t>0$ and $\mu>0$, we have $t=\pi \mu$, and then $B$ must be diagonal. Hence

$$
R A \star g(z)=c^{2} z^{\mathrm{i} t / \pi} \frac{\xi_{2}(z)}{\xi_{1}(z)}, \quad B=\left(\begin{array}{cc}
c & 0 \\
0 & c^{-1}
\end{array}\right)
$$

proving the assertion.

\section{Monodromy of punctured hyperbolic metrics}

We consider a conformal metric $d \sigma^{2}$ on $\Delta^{*}$ of constant Gaussian curvature -1 , called a punctured hyperbolic metric. Then there exists a meromorphic function $g: \widetilde{\Delta}^{*} \rightarrow \boldsymbol{C} \cup\{\infty\} \backslash\{|z|=1\}$ such that

$$
d \sigma^{2}=\frac{4|d g|^{2}}{\left(1-|g|^{2}\right)^{2}}
$$

which is called the developing map of $d \sigma^{2}$. Since $d \sigma^{2}$ is a well defined hyperbolic metric on $\Delta^{*}$, either $|g|<1$ or $|g|>1$ holds on $\Delta^{*}$.

We remark that, for a CMC-1 immersion $f: \Delta^{*} \rightarrow S_{1}^{3}$, the metric $d \sigma^{2}$ as in (1.15) is an example of a hyperbolic metric, and the secondary Gauss map is a developing map of it.

The developing map $g$ is not unique, and the set of all developing maps of $d \sigma^{2}$ coincides with

$$
\left\{A \star g ; A \in \mathrm{SU}_{1,1}\right\} \cup\left\{A \star \frac{1}{g}=A\left(\begin{array}{cc}
0 & \mathrm{i} \\
\mathrm{i} & 0
\end{array}\right) \star g ; A \in \mathrm{SU}_{1,1}\right\} .
$$

Set

$$
S\left(d \sigma^{2}\right):=S(g)=\left(w_{z z}-\frac{\left(w_{z}\right)^{2}}{2}\right) d z^{2}
$$

where $d \sigma^{2}=\mathrm{e}^{w}|d z|^{2}$, that is, $w:=\log \left(4\left|g_{z}\right|^{2} /\left(1-|g|^{2}\right)^{2}\right)$. We call the projective connection $S\left(d \sigma^{2}\right)$ the Schwarzian derivative of $d \sigma^{2}$. Since the metric $d \sigma^{2}$ is well defined on $\Delta^{*}$, the developing map $g$ is a $\mathrm{PSU}_{1,1}$-lift of the $\mathrm{PSU}_{1,1}$-projective connection $S\left(d \sigma^{2}\right)$.

If $g$ is a developing map of $d \sigma^{2}=K_{d s^{2}} d s^{2}$ in (1.15), then

$$
g \circ \tau^{-1}=T \star g \quad \text { for some } T \in \mathrm{PSU}_{1,1} .
$$

If the matrix $T$ is elliptic (resp. parabolic, hyperbolic), the metric $d \sigma^{2}$ is said to have elliptic (resp. parabolic, hyperbolic) monodromy. 
Definition 2.1. We say that a hyperbolic punctured metric $d \sigma^{2}$ has a regular singularity at the origin if $S\left(d \sigma^{2}\right)$ has a regular singularity at the origin, that is, it has at most a pole of order 2 .

Theorem 2.1. Any conformal hyperbolic metric on $\Delta^{*}$ has a regular singularity at $z=0$.

Proof. Let $g$ be a developing map of a conformal hyperbolic metric $d \sigma^{2}$ on $\Delta^{*}$.

Suppose $d \sigma^{2}$ has elliptic monodromy. Since $d \sigma^{2}$ has no singular points on $\Delta^{*},|g|<1$ or $|g|>1$ holds on $\Delta^{*}$. Since $1 / g$ is also a developing map of $d \sigma^{2}$, we may assume that $|g|<1$. By Proposition 2.1, there exists a real number $\mu$ such that $h(z):=z^{-\mu} g(z)$ is a single-valued function on $\Delta^{*}$. Multiplying $h(z)$ by $z^{k}(k \in \boldsymbol{Z})$, we may assume that $-1<\mu \leq 0$ without loss of generality. Thus

$$
|h(z)|=|z|^{-\mu}|g(z)|<|z|^{-\mu}<1,
$$

and $h(z)$ has more than two exceptional values, so has at most a pole at $z=0$, by the Great Picard theorem. Then by (1) (ii) in Proposition 2.1, $S(g)$ has a regular singularity at the origin.

Suppose $d \sigma^{2}$ has parabolic monodromy. Applying Proposition 2.1 for the $\mathrm{PSU}_{1,1}$-projective connection $S\left(d \sigma^{2}\right)$ with $\varepsilon=-1$ and $t=\pi$, we can take a $\mathrm{PSU}_{1,1}$-lift $g$ such that

$$
h=\hat{g}+\mathrm{i} \log z \quad\left(\hat{g}(z):=R \star g(z)=\frac{1}{\mathrm{i}} \frac{g(z)+1}{g(z)-1}\right)
$$

is a single-valued meromorphic function on $\Delta^{*}$, where $R$ is the matrix in (2.2). Since $d \sigma^{2}$ has no singular points on $\Delta^{*},|g|>1$ or $|g|<1$ holds. In particular, because $z \mapsto R \star z$ maps the unit disk into the upper-half plane, we have $\operatorname{Im} \hat{g}>0($ resp. $\operatorname{Im} \hat{g}<0)$ if $|g|<1$ (resp. $|g|>1)$. Here, it holds that

$$
|z \exp (\mathrm{i} h)|=|\exp (\mathrm{i} \hat{g})|=\exp (-\operatorname{Im} \hat{g}) .
$$

Thus,

$$
\begin{array}{rlrl}
|z \exp (\mathrm{i} h)| & =\exp (-\operatorname{Im} \hat{g})<1 & & \text { (if }|g|<1), \\
\left|\frac{1}{z} \exp (-\mathrm{i} h)\right| & =\exp (\operatorname{Im} \hat{g})<1 & (\text { if }|g|>1) .
\end{array}
$$

Thus by the Great Picard theorem, there exist an integer $m$ and a holomorphic function $\varphi(z)$ with $\varphi(0) \neq 0$ such that $\exp ( \pm \mathrm{i} h(z))=z^{m} \varphi(z)$, that is,

$$
\pm \mathrm{i} h(z)=m \log z+\log \varphi(z) .
$$


Since $h(z)$ is single valued, $m$ must be 0 . Therefore, $h(z)$ can be extended to be holomorphic at $z=0$, and then by (2)(ii) of Proposition 2.1, the origin is a regular singularity of $S\left(d \sigma^{2}\right)$.

To prove the hyperbolic case, we need the following

Fact 2.1 (Montel's theorem). If a family of holomorphic functions $\left\{f_{n}\right\}_{n=1,2,3, \ldots}$ defined on a domain $D(\subset \boldsymbol{C})$ have two exceptional values in common, then they are a normal family, that is, there is a subsequence $\left\{f_{n_{j}}\right\}_{j=1,2,3, \ldots}$ such that either $\left\{f_{n_{j}}\right\}_{j=1,2,3, \ldots}$ or $\left\{1 / f_{n_{j}}\right\}_{j=1,2,3, \ldots}$ converges uniformly on every compact set in $D$.

Proof of Theorem 2.1, continued. The proof for the hyperbolic case is parallel to the proofs of Propositions 4 and 5 in [3].

Suppose $d \sigma^{2}$ has hyperbolic monodromy. Again, we may assume that $|g|<1$ without loss of generality. By Proposition 2.1, again replacing $g$ by $A \star g$ for some $A \in \mathrm{SU}_{1,1}$ if necessary, there exists a positive real number $\mu$ such that $h(z):=z^{-\mathrm{i} \mu}(R \star g(z))$ is a single-valued meromorphic function on $\Delta^{*}$. The function

$$
\hat{g}(z):=R \star g(z)=\frac{1}{\mathrm{i}} \frac{g(z)+1}{g(z)-1}
$$

has neither zeros nor poles in $\Delta^{*}$, and $\operatorname{Im} \hat{g}>0$. We now define a set

$$
\Omega:=\left\{z \in C ; 0<|z|<1,|\arg z|<\frac{2 \pi}{3}\right\}
$$

and analytic functions $\zeta$ and $f_{n}$ for $n=1,2,3, \ldots$ from $\Omega$ to $\boldsymbol{C}$ by $\zeta(z):=$ $\hat{g}\left(z^{2}\right)$ and $f_{n}(z):=\hat{g}\left(z^{2} / 2^{2 n}\right)$. Then $\left\{f_{n}\right\}_{n=1}^{\infty}$ is a family of holomorphic functions on $\Omega$. Since $\operatorname{Im} \hat{g}>0$, we have $\operatorname{Im} f_{n}>0$. Thus $\left\{f_{n}\right\}$ is a normal family by Montel's theorem. Two possible cases arise.

Case 1. First we consider the case that a subsequence $\left\{f_{n}\right\}$ converges to a holomorphic function uniformly on any compact subset of $\Omega$. Since

$$
\begin{aligned}
\Omega_{l}:= & \left\{z \in \boldsymbol{C} ;|z|=\frac{1}{2^{l}},|\arg z| \leq \frac{3}{5} \pi\right\} \cup\left\{z \in \boldsymbol{C} ;|z|=\frac{1}{2^{l+1}},|\arg z| \leq, \frac{3}{5} \pi\right\} \\
& \cup\left\{z \in \boldsymbol{C} ;|\arg z|=\frac{3}{5} \pi, \frac{1}{2^{l+1}} \leq|z| \leq \frac{1}{2^{l}}\right\}
\end{aligned}
$$

for a positive integer $l \in Z_{+}$is a compact subset of $\Omega$, there exist a positive number $M \in \boldsymbol{R}_{+}$and a positive integer $n_{0} \in \boldsymbol{Z}_{+}$such that $\left|f_{n}(z)\right|<M$ holds 
on $\Omega_{1}$ for $n \geq n_{0}$. This implies that $|\zeta(z)|<M$ on $\Omega_{n+1}$ for $n \geq n_{0}$. Then by the maximum principle, we have

$$
|\zeta(z)|<M \quad \text { on } \quad\left\{z \in C ; \frac{1}{2^{n+1}} \leq|z| \leq \frac{1}{2^{n}},|\arg z| \leq \frac{3}{5} \pi\right\}
$$

for each $n>n_{0}+1$. Thus we have

$$
\left|\hat{g}\left(z^{2}\right)\right|=|\zeta(z)|<M \quad \text { on } \quad\left\{z \in C ; 0<|z| \leq \frac{1}{2^{n_{0}+1}},|\arg z| \leq \frac{3}{5} \pi\right\} .
$$

On the other hand, since $\mathrm{e}^{-\pi|\mu|}<\left|z^{-\mathrm{i} \mu}\right|<\mathrm{e}^{\pi|\mu|}$ for $|z|<1$ and $|\arg z|<\pi$, the function $h(z)$ is bounded in a punctured neighborhood of $z=0$ and has a removable singularity there.

Case 2. Next, we consider the case that a subsequence $\left\{1 / f_{n}\right\}$ converges to a holomorphic function $f$. Then we can conclude that $1 / h(z)$ is bounded on $\Delta^{*}$. In this case $h(z)$ has at most a pole at the origin.

In both cases, $S(g)=S\left(R^{-1} \star\left(z^{\mathrm{i} \mu} h(z)\right)\right)$ has at most a pole of order two at $z=0$.

Remark 2.3. In Corollary 3.1, we shall show that in fact the monodromy of $d \sigma^{2}$ can never be hyperbolic.

\section{Intrinsic behavior of regular ends}

Let $f: M^{2} \rightarrow S_{1}^{3}$ be a weakly complete CMC-1 face of finite topology, and let $\bar{M}^{2}$ be a compact Riemann surface such that $M^{2}$ is diffeomorphic to $\bar{M}^{2} \backslash\left\{p_{1}, \ldots, p_{n}\right\}$.

Definition 3.1. A puncture-type end $p_{j}$ of $f$ is called regular if the hyperbolic Gauss map $G$ has at most a pole at $p_{j}$.

Definition 3.2. On the other hand, we say a puncture-type end $p_{j}$ is $g$ regular if the Schwarzian derivative $S(g)$ of the secondary Gauss map $g$ has at most a pole of order 2 at $p_{j}$, that is, the pseudometric $d \sigma^{2}$ in (1.15) has a regular singularity at $p_{j}$ (cf. Definition 2.1 ).

When $g$ is single-valued, $g$-regularity implies that $g$ has at most a pole at the end. When the Hopf differential has at most a pole of order 2, regularity and $g$-regularity are equivalent, by (1.20). 
Theorem 2.1 can now be stated in terms of CMC-1 faces as follows:

Lemma 3.1. All ends of a complete CMC-1 face are g-regular.

Proof. By Proposition 1.1, all ends are of puncture-type. So we can set $M^{2}=\bar{M}^{2} \backslash\left\{p_{1}, \ldots, p_{n}\right\}$, where $\bar{M}^{2}$ is a compact Riemann surface. Let $(g, \omega)$ be a Weierstrass data for $f$. Since the singular set is compact, the metric $d \sigma^{2}$ as in (1.15) is a punctured hyperbolic metric in a punctured neighborhood of $p_{j}$. Then $d \sigma^{2}$ has a regular singularity by Theorem 2.1, and hence $f$ is $g$-regular at $p_{j}$.

Definition 3.3. An elliptic end of a CMC-1 face is integral if the monodromy of the secondary Gauss map is the identity, and non-integral otherwise.

Lemma E1. Let $f: \Delta^{*} \rightarrow S_{1}^{3}$ be a g-regular non-integral elliptic end. Then the singular set does not accumulate at the end 0 .

Proof. One can take the secondary Gauss map $g$ to be $g(z)=z^{\mu} h(z)$ on a neighborhood of the end, where $\mu \in \boldsymbol{R} \backslash \boldsymbol{Z}$ and $h(z)$ is holomorphic at the end $z=0$ with $h(0) \neq 0$. Since $\mu \neq 0$, the singular set $\{|g|=1\}$ cannot accumulate at the origin.

On the other hand, an integral elliptic end might or might not be complete:

Example 3.1. For non-zero integers $m$ and $n$ with $|m| \neq|n|$, we set $g=$ $1-z^{m}$ and $G=z^{n}$. Setting $Q=(S(g)-S(G)) / 2$ and $\omega=Q / d g$, we see that (1.5) gives a Riemannian metric on $C \backslash\{0\}$. So using Small's formula (1.10), we have a CMC-1 face defined on $\boldsymbol{C} \backslash\{0\}$ with integral elliptic ends at $z=0, \infty$. The singular set is

$$
\left\{z \in \boldsymbol{C} \backslash\{0\} ;|z|^{2 m}-2 \operatorname{Re}\left(z^{m}\right)=0\right\}
$$

(see Figure 1 for the case $m=3$ ). Thus the singular set accumulates at $z=0$ but not at $z=\infty$. Thus $z=\infty$ is a complete integral elliptic end, but $z=0$ is an incomplete integral elliptic end.

To state the behavior of an incomplete (integral) elliptic end, we introduce a notation: For an $m \in \boldsymbol{Z}_{+}$, an $\varepsilon \in(0, \pi /(2 m))$ and a $\delta \in[0, \pi / m]$, we 


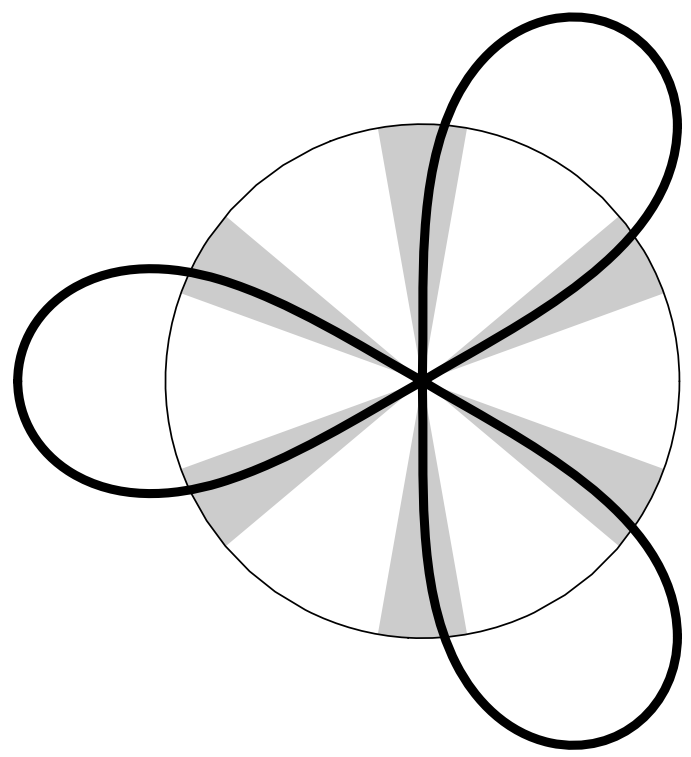

Figure 1: The thick curve is the singular set of Example 3.1 for the case $m=3$. The shaded parts indicate the set $S(3, \varepsilon, \pi / 6)$.

define the open subset (which is a union of sectors, see Figure 1)

$$
S(m, \varepsilon, \delta):=\bigcup_{k=0}^{2 m-1}\left\{z \in C \backslash\{0\} ; \frac{k}{m} \pi+\delta-\varepsilon<\arg z<\frac{k}{m} \pi+\delta+\varepsilon\right\} .
$$

Lemma E2. Suppose $f: \Delta^{*} \rightarrow S_{1}^{3}$ is a g-regular integral elliptic end. If the singular set accumulates at the end, then there are an $m \in \boldsymbol{Z}_{+}$and $a \delta \in \boldsymbol{R}$ such that, for any $\varepsilon>0$, there exists an $r>0$ so that the singular set of $f$ in $\{z ; 0<|z|<r\}$ lies in $S(m, \varepsilon, \delta)$.

Proof. The assertion of the lemma does not depend on a choice of the complex coordinate at the origin.

Since the singular set accumulates at 0 , we have $|g(0)|=1$. Then by Proposition 2.1(1), $g(z)$ is holomorphic at $z=0$. Moreover, we may set $g(0)=1$. Then $\varphi(z):=\log g(z)$ is well defined on a neighborhood of $z=0$ and $\varphi(0)=0$. Using the Weierstrass preparation theorem, we may further assume, without loss of generality, that $\varphi(z)=z^{m}$ for some positive integer $m$. Here, $|g(z)|=1$ is equivalent to $\operatorname{Re} \varphi(z)=0$. Thus, the singular set is expressed as $\{\cos m \theta=0\}$, where $z=r \mathrm{e}^{\mathrm{i} \theta}$. 
Definition 3.4. A parabolic end of a CMC-1 face is of the first kind if

$$
S\left(d \sigma^{2}\right)-\frac{d z^{2}}{2 z^{2}}=S(g)-\frac{d z^{2}}{2 z^{2}}=S(G)+2 Q-\frac{d z^{2}}{2 z^{2}}
$$

has at most a pole of order 1. Otherwise, it is of the second kind.

Lemma P. Let $f: \Delta^{*} \rightarrow S_{1}^{3}$ be a g-regular parabolic end. If the end is of the first kind, the singular set does not accumulate at the end. If the end is of the second kind, then the singular set does accumulate at the end. In this case, there exist an $m \in Z_{+}$and $a \delta(\delta \in[0, \pi / m])$ such that, for all $\varepsilon>0$, there exists an $r>0$ so that the singular set of $f$ in $\{z ; 0<|z|<r\}$ lies in $S(m, \varepsilon, \delta)$.

Proof. Let $g$ be the secondary Gauss map. Since the end is parabolic, the Schwarzian derivative $P:=S(g)$ determines a $\mathrm{PSU}_{1,1}$-projective connection of parabolic monodromy. Then by (2) in Proposition 2.1 for $\varepsilon=-1$ and $t=\pi$, there exists a $\mathrm{PSU}_{1,1}$ lift $g_{0}$ such that

$$
h(z)=\hat{g}_{0}(z)+\mathrm{i} \log z \quad\left(\hat{g}_{0}(z)=R \star g_{0}(z)=\frac{1}{\mathrm{i}} \frac{g_{0}(z)+1}{g_{0}(z)-1}\right)
$$

is a meromorphic function on $\Delta^{*}$. Here, there exists a matrix $A \in \mathrm{SU}_{1,1}$ such that $g=A \star g_{0}$ or $1 / g=A \star g_{0}$ holds. Thus, by the $\mathrm{SU}_{1,1}$-ambiguity of the secondary Gauss map, we may assume $g=g_{0}$ or $1 / g_{0}$. Moreover, replacing $f$ with $-f$ if necessary (see Remark 1.9), we may assume $g=g_{0}$ without loss of generality.

Since the end is $g$-regular, (2)(ii) of Proposition 2.1 yields that $h(z)$ is meromorphic at $z=0$. Thus, we can write

$$
\hat{g}(z)=\hat{g}_{0}(z)=-\mathrm{i} \log z+z^{m} \varphi(z) \quad(\varphi(0) \neq 0, m \in Z)
$$

where $\varphi(z)$ is a holomorphic function on a neighborhood of the origin. Then there exist an $a \in \boldsymbol{R} \backslash\{0\}$ and a $\gamma \in(-\pi, \pi)$ such that

$$
\operatorname{Im} \hat{g}(z)=-\log r+a r^{m} \sin (m \theta+\gamma)+o\left(r^{m+1}\right), \quad z=r \mathrm{e}^{\mathrm{i} \theta}
$$

Here, the singular set $\{|g|=1\}$ is written as $\{\operatorname{Im} \hat{g}=0\}$. 

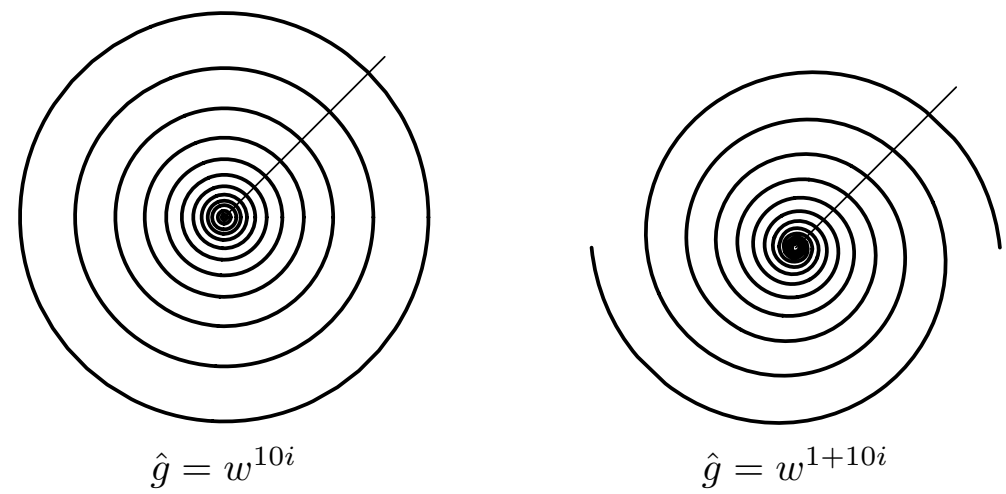

Figure 2: The thick curves indicate the singular sets of hyperbolic ends with secondary Gauss map $g=R^{-1} \star \hat{g}$, as discussed in Lemma H. The thin lines indicate rays in $\Delta^{*}$ emanating from the origin. Here we see that the singular sets intersect rays emanating from the origin infinitely many times.

If the end is of the first kind, then $m \geq 0$ by (2) (iii) in Proposition 2.1. Therefore, for each fixed $\theta$, the right-hand side approaches $\infty$ as $r \rightarrow 0$, which implies that the singular points do not accumulate at the end.

If the end is of the second kind, then $m<0$. Therefore, for each fixed $\theta$, the right-hand side approaches $\infty$ if $a \sin (m \theta+\gamma)>0$ and $-\infty$ if $a \sin (m \theta+$ $\gamma)<0$ as $r \rightarrow 0$, giving solutions of $\operatorname{Im} \hat{g}(z)=0$ for sufficiently small $r$ near the lines $\sin (m \theta+\gamma)=0$. This implies the second assertion.

Lemma H. Let $f: \Delta^{*} \rightarrow S_{1}^{3}$ be a g-regular hyperbolic end. Then any ray in $\Delta^{*}$ emanating from the origin meets the singular set infinitely many times. (See Figure 2.)

Remark 3.1. This intersection property does not depend on the choice of a complex coordinate for a punctured neighborhood of the end.

Proof of Lemma $H$. By Proposition 2.1 and an appropriate choice of $g$, if we set $\hat{g}=R \star g$, then there is a $\mu \in \boldsymbol{R} \backslash\{0\}$ such that

$$
h(z):=z^{-\mathrm{i} \mu} \hat{g}(z)
$$

is a meromorphic function on $\Delta^{*}$. Since $f$ is $g$-regular, (3)(ii) of Proposition 2.1 implies $h$ has at most a pole at the origin, and $S(g)$ has a pole of 
order exactly 2 at $z=0$. Thus we can rewrite

$$
\hat{g}(z)=z^{m+\mathrm{i} \mu} \varphi(z) \quad(\varphi(0) \neq 0, m \in \boldsymbol{Z}),
$$

where $\varphi(z)$ is a single-valued holomorphic function on $\Delta=\Delta^{*} \cup\{0\}$. Now, we set

$$
w=z \exp \left(\frac{\log \varphi}{m+\mathrm{i} \mu}\right),
$$

which gives a new coordinate $w$ around the end, now at $w=0$. Then $\hat{g}(w)=$ $w^{m+\mathrm{i} \mu}$. Since $g=(\hat{g}-\mathrm{i}) /(\hat{g}+\mathrm{i})$, setting $w=r \mathrm{e}^{\mathrm{i} \theta}$, the singular set is

$$
\begin{aligned}
\{w ;|g(w)|=1\} & =\{w ; \operatorname{Im}(\hat{g}(w))=0\} \\
& =\{(r, \theta) \in(0,1) \times(-\pi, \pi) ; \mu \log r+m \theta \equiv 0 \quad(\bmod \pi)\},
\end{aligned}
$$

that is, $r=\exp ((n \pi-m \theta) / \mu), n \in \boldsymbol{Z}$, which is a log-spiral when $m \neq 0$. If $m=0$, the singular set is a union of infinitely many disjoint circles. In any case, the singular set meets any ray based at $w=0$ infinitely many times. (See Figure 2, left-hand side for the case $m=0$ and right-hand side for the case $m \neq 0$.)

Corollary 3.1. The monodromy of a hyperbolic metric on $\Delta^{*}$ is either elliptic or parabolic. That is, hyperbolic monodromy never occurs.

Proof. Suppose that a hyperbolic metric $d \sigma^{2}$ on $\Delta^{*}$ has hyperbolic monodromy. Let $g$ be a developing map for $d \sigma^{2}$. The data $(g, \omega=d z)$ produces an $F$ as in (1.6), and then the immersion $f=F e_{3} F^{*}: \Delta^{*} \rightarrow S_{1}^{3}$ is without singularities, since $d \sigma^{2}$ is non-singular. By Theorem 2.1, $f$ is $g$-regular at $z=0$. Then by Lemma $H$, the singularities accumulate at the end, a contradiction.

Lemmas E1, E2, $\mathrm{P}$ and $\mathrm{H}$ imply:

Corollary 3.2 (Characterization of hyperbolic ends). A g-regular end $f: \Delta^{*} \rightarrow S_{1}^{3}$ of a CMC-1 face is hyperbolic if and only if every ray in $\Delta^{*}$ emanating from the origin meets the singular set infinitely many times.

\section{Completeness}

We now give two theorems on complete CMC-1 faces.

Theorem 3.1. Any complete end of a CMC-1 face is either g-regular elliptic or g-regular parabolic of the first kind. 
Proof. By Proposition 1.1, the end is of puncture-type. Moreover, Theorem 2.1 implies the end is $g$-regular. Thus the theorem follows from Corollary 3.1 and Lemma P.

Theorem 3.2. Any complete CMC-1 face is of finite type. (The definition of finite type is given in Definition 1.4.)

Proof. Let $f: M^{2} \rightarrow S_{1}^{3}$ be a complete CMC-1 face. Then by Proposition 1.1, there is a compact Riemann surface $\bar{M}^{2}$ such that $M^{2}$ is biholomorphic to $\bar{M}^{2} \backslash\left\{p_{1}, \ldots, p_{n}\right\}$.

We fix any end $p_{j}$, and take a small coordinate neighborhood $(U, z)$ with $z=0$ at $p_{j}$. We may assume that there are no singular points on $U \backslash\left\{p_{j}\right\}$, and thus we may also assume that $|g|<1$ on $U \backslash\left\{p_{j}\right\}$ for a secondary Gauss map $g$. We know from Theorem 3.1 that the end is a $g$-regular elliptic end or a $g$-regular parabolic end of the first kind.

First, we consider the elliptic case. Since $|g|<1$, we may assume that there exist some $\mu(\geq 0)$ and a holomorphic function $h(z)$ on $U$ with $h(0) \neq 0$ such that $g(z)=z^{\mu} h(z)$. If $|g(0)|=1$, then $\mu=0$ and

$$
g(z)=\mathrm{e}^{\mathrm{i} \theta}\left(1+a z^{m}+o\left(z^{m}\right)\right)
$$

for some $\theta \in \boldsymbol{R}, a \in \boldsymbol{C} \backslash\{0\}$ and $m \in \boldsymbol{Z}_{+}$, which contradicts the fact that $|g|<1$ on $U \backslash\left\{p_{j}\right\}$. Hence $|g(0)|<1$. Therefore, there exist a neighborhood $\widetilde{U} \subset U$ of $p_{j}$ and an $\varepsilon>0$ such that $|g|^{2}<1-\varepsilon$ on $\widetilde{U}$. So, on $\widetilde{U}$,

$$
K_{d s^{2}} d s^{2}=\frac{4|d g|^{2}}{\left(1-|g|^{2}\right)^{2}} \leq \frac{4}{\varepsilon^{2}} \frac{4|d g|^{2}}{\left(1+|g|^{2}\right)^{2}}=\frac{4}{\varepsilon^{2}}\left(-K_{d \hat{s}^{2}}\right) d \hat{s}^{2},
$$

where $d \hat{s}^{2}$ is the metric as in (1.5), which is defined on $\widetilde{U} \backslash\left\{p_{j}\right\}$ because $g(z)=z^{\mu} h(z)$. Since $p_{j}$ is a regular singularity of the punctured spherical metric

$$
d \hat{\sigma}^{2}:=\left(-K_{d \hat{s}^{2}}\right) d \hat{s}^{2}=\frac{4|d g|^{2}}{\left(1+|g|^{2}\right)^{2}},
$$

$d \hat{\sigma}^{2}$ has finite area, so $d s^{2}$ has finite total curvature on $\widetilde{U} \backslash\left\{p_{j}\right\}$, by (3.1).

Next we consider the parabolic case. By Theorem 3.1, the end is parabolic of the first kind. Then without loss of generality, we may assume there exists a holomorphic function $h(z)$ on $\Delta$ such that (we set $t=\pi$ and replace $h(z)$ 
by $\mathrm{i} h(z)$ in the proof of Proposition 2.1)

$$
\hat{g}(z)=\mathrm{i}(h(z) \pm \log z), \quad \text { where } \quad \hat{g}(z)=R \star g(z)=\frac{1}{\mathrm{i}} \frac{g(z)+1}{g(z)-1} .
$$

If we set $k(z):=h(z) \pm \log z+1$, we have $g=1-2 / k, g^{\prime}=2 k^{\prime} / k^{2}$ and

$$
1-|g(z)|^{2}=\frac{4(\operatorname{Re} k(z)-1)}{|k(z)|^{2}}=\frac{4(\operatorname{Re} h(z) \pm \operatorname{Re} \log z)}{|k(z)|^{2}} .
$$

So we have

$$
d \sigma^{2}=\frac{4|d g|^{2}}{\left(1-|g|^{2}\right)^{2}}=\frac{\left|h^{\prime}(z) \pm(1 / z)\right|^{2}|d z|^{2}}{(\operatorname{Re} h(z) \pm \operatorname{Re} \log z)^{2}}
$$

We set $c:=\sup _{z \in \Delta}|\operatorname{Re} h(z)|$ and $r=|z|$. Since $\log r \rightarrow-\infty$ as $z \rightarrow 0$, we may assume $-\log r>c$. Then

$$
|\operatorname{Re} h(z) \pm \log r| \geq|| \operatorname{Re} h(z)|-| \log r|| \geq|c+\log r|, \quad \text { and }
$$

where $C=\sup _{z \in \Delta}\left|z h^{\prime}(z)+1\right|$. Since

$$
\int_{0}^{\varepsilon} \frac{C^{2} r d r}{r^{2}(c+\log r)^{2}}=-\frac{C^{2}}{c+\log \varepsilon}<\infty
$$

the area of a sufficiently small punctured neighborhood of $z=0$ with respect to $d \sigma^{2}$ is finite, which proves the assertion.

Theorem 0.1 in the introduction follows from Theorems 3.1 and 3.2.

\section{The light-cone Gauss map and extrinsic behavior of ends}

Let $\mathrm{LC}=\left\{x \in \boldsymbol{R}_{1}^{4} ;\langle x, x\rangle=0\right\}$ be the light-cone of $\boldsymbol{R}_{1}^{4}$, with future and past light cones

$$
\mathrm{LC}_{ \pm}:=\left\{x=\left(x_{0}, x_{1}, x_{2}, x_{3}\right) \in \mathrm{LC} ; \pm x_{0}>0\right\}
$$

The multiplicative group $\boldsymbol{R}_{+}$of the positive real numbers acts on $\mathrm{LC}_{ \pm}$by scalar multiplication. The ideal boundary $\partial S_{1}^{3}$ of $S_{1}^{3}$ consists of two (future 
and past) components

$$
\partial_{ \pm} S_{1}^{3}:=\mathrm{LC}_{ \pm} / \boldsymbol{R}_{+}
$$

each of which are identified with $\boldsymbol{C} \cup\{\infty\}$ by the projection

$$
\pi: \partial_{ \pm} S_{1}^{3} \ni\left[\left(v_{0}, v_{1}, v_{2}, v_{3}\right)\right] \longmapsto \frac{1}{v_{0}-v_{3}}\left(v_{1}+\mathrm{i} v_{2}\right) \in \boldsymbol{C} \cup\{\infty\}
$$

The isometries of $\boldsymbol{R}_{1}^{4}$ induce Möbius transformations on $\boldsymbol{C} \cup\{\infty\}$. The boundary $\partial S_{1}^{3}$ is identified with the set of equivalence classes of oriented time-like geodesics in $S_{1}^{3}$.

In particular, for a space-like immersion $f: M^{2} \rightarrow S_{1}^{3}$ with the (timelike) unit normal vector field $\nu$, the equivalence class $[f+\nu]$ determines a point in $\partial S_{1}^{3}$ for each $p \in M^{2}$. Hence we have the light-cone Gauss map

$$
L=[f+\nu]: M^{2} \longrightarrow \partial S_{1}^{3}
$$

Let $f: M^{2} \rightarrow S_{1}^{3}$ be a CMC-1 face, and $p \in M^{2}$ a regular point, that is, $f$ is an immersion in a neighborhood of $p$. Under the identification of $\boldsymbol{R}_{1}^{4}$ and $\operatorname{Herm}(2)$ as in (1.1), we can compute that the unit normal vector $\nu$ is

$$
\nu=\frac{1}{|g|^{2}-1} F\left(\begin{array}{cc}
1+|g|^{2} & 2 g \\
2 \bar{g} & 1+|g|^{2}
\end{array}\right) F^{*},
$$

where $F$ is the holomorphic lift of $f$ and $g$ is the secondary Gauss map. Hence

$$
L=\left[\frac{2}{|g|^{2}-1} F\left(\begin{array}{cc}
|g|^{2} & g \\
\bar{g} & 1
\end{array}\right) F^{*}\right]=\operatorname{sgn}\left(|g|^{2}-1\right)\left[F\left(\begin{array}{cc}
|g|^{2} & g \\
\bar{g} & 1
\end{array}\right) F^{*}\right]
$$

where $\operatorname{sgn}\left(|g|^{2}-1\right)$ is the sign of the function $|g|^{2}-1$. Thus, we have:

Proposition 4.1. The light-cone Gauss map L of a CMC-1 face takes values in $\partial_{+} S_{1}^{3}$ (resp. $\left.\partial_{-} S_{1}^{3}\right)$ if $|g|>1$ (resp. $|g|<1$ ). Moreover, its projection $\pi \circ L$ is the hyperbolic Gauss map $G$ as in (1.9), which extends to the singular set.

Proof. By (4.2), the $x_{0}$-component of $f+\nu$ is $\frac{1}{|g|^{2}-1} \operatorname{trace}\left(F\left(\begin{array}{cc}|g|^{2} & g \\ \bar{g} & 1\end{array}\right) F^{*}\right)=\frac{1}{|g|^{2}-1}\left(\left|g F_{11}+F_{12}\right|^{2}+\left|g F_{21}+F_{22}\right|^{2}\right)$, 
where $F=\left(F_{i j}\right)_{i, j=1,2}$. Here, $\left|g F_{11}+F_{12}\right|^{2}+\left|g F_{21}+F_{22}\right|^{2}>0$ holds because $\operatorname{det} F=1 \neq 0$, implying the first part of the proposition. By (4.2), (4.1), (1.6) and (1.9), we have

$$
\pi \circ L=\pi \circ[f+\nu]=\frac{g F_{11}+F_{12}}{g F_{21}+F_{22}}=\frac{d F_{11}}{d F_{21}}=G,
$$

and this completes the proof.

Next, we give a criterion for when a complete regular end approaches $\partial_{+} S_{1}^{3}$ or $\partial_{-} S_{1}^{3}$ :

Proposition 4.2. Let $f: \Delta^{*} \rightarrow S_{1}^{3}$ be a complete regular end at $z=0$ and let $g$ be a secondary Gauss map of $f$. Then the image of $f$ converges to a point in $\partial_{+} S_{1}^{3}\left(\right.$ resp. $\left.\partial_{-} S_{1}^{3}\right)$ at the end if and only if $|g|<1 \quad($ resp. $|g|>1)$ near the end.

Proof. We can change the holomorphic null lift $F$ to $F^{\natural}$ as in (1.14), so that $f$ and its secondary Gauss map $g$ change to $-f$ and $1 / g$. The end of $f$ approaches $\partial_{ \pm} S_{1}^{3}$ if and only if $-f$ approaches $\partial_{\mp} S_{1}^{3}$, so it is sufficient to prove this result under the assumption $|g|<1$ on $\Delta^{*}$. By Theorem 3.1, the end is either elliptic or parabolic.

First we assume the end is elliptic. Replacing $F$ by $a F b^{-1}\left(a \in \mathrm{SL}_{2} \boldsymbol{C}\right.$, $\left.b \in \mathrm{SU}_{1,1}\right)$ and using the Weierstrass preparation theorem if necessary, we may assume without loss of generality that the hyperbolic and secondary Gauss maps are

$$
G(z)=z^{m}, \quad g(z)=z^{\mu} h(z) \text { for some } m \in Z_{+}, \mu \in \boldsymbol{R} \backslash\{0\},
$$

where $h$ is a holomorphic function on $\Delta$ with $h(0) \neq 0$. Here $\mu>0$ because $|g|<1$.

If $m \neq \mu$, Small's formula (1.10) implies that

$$
F=\frac{1}{2 \sqrt{m \mu}}\left(\begin{array}{rr}
-z^{\frac{m-\mu}{2}}(m+\mu)(1+o(1)) & z^{\frac{m+\mu}{2}}(m-\mu)(1+o(1)) \\
z^{\frac{-m-\mu}{2}}(m-\mu)(1+o(1)) & -z^{\frac{-m+\mu}{2}}(m+\mu)(1+o(1))
\end{array}\right)
$$

Since $m$ and $\mu$ are positive, the first component $x_{0}$ is

$$
x_{0}=\frac{1}{2} \operatorname{trace}\left(F e_{3} F^{*}\right)=\frac{(m-\mu)^{2}}{8 m \mu} r^{-m-\mu}(1+o(1)) \rightarrow+\infty \quad(r \rightarrow 0),
$$


where $z=r \mathrm{e}^{\mathrm{i} \theta}$. Other components of $f=\left(x_{0}, x_{1}, x_{2}, x_{3}\right)$ are expressed as

$$
\begin{aligned}
x_{1}+\mathrm{i} x_{2} & =\mathrm{e}^{\mathrm{i} m \theta} r^{-\mu} \frac{\mu^{2}-m^{2}}{4 \mu m}(1+o(1)), \\
x_{3} & =-\frac{(m-\mu)^{2}}{8 m \mu} r^{-m-\mu}(1+o(1)) .
\end{aligned}
$$

We now consider the stereographic projection given in [7]:

$$
\begin{aligned}
& \Pi:\left\{\left(x_{0}, x_{1}, x_{2}, x_{3}\right) \in S_{1}^{3} ; x_{0}>1\right\} \ni\left(x_{0}, x_{1}, x_{2}, x_{3}\right) \\
& \longmapsto \frac{1}{1+x_{0}}\left(x_{1}, x_{2}, x_{3}\right) \in\left\{\left(X_{1}, X_{2}, X_{3}\right) \in \boldsymbol{R}^{3} ; \frac{1}{2}<\sum_{j=1}^{3}\left(X_{j}\right)^{2}<1\right\}
\end{aligned}
$$

which is a diffeomorphism. Then $\Pi \circ f$ is expressed as

$$
\Pi \circ f=(0,0,-1)+o(1) .
$$

Thus, $\Pi \circ f$ approaches $(0,0,-1) \in S^{2}=\partial_{+} S_{1}^{3}$.

When $\mu=m$, by (1.10) again, $F_{11}, F_{12}$ and $F_{22}$ are bounded on a neighborhood of 0 , and these components can be extended to become holomorphic on a neighborhood of 0 . If $F_{21}$ is bounded, $F$ must be holomorphic and then the induced metric is bounded, which contradicts the weak completeness of the end. Hence $F_{21}$ has a pole at 0 . So the $x_{0}$-component of $f$ is

$$
x_{0}=\frac{1}{2}\left|F_{21}\right|^{2}+(\text { a bounded function }) \rightarrow+\infty \quad(z \rightarrow 0) .
$$

Moreover, since

$$
\begin{aligned}
x_{3} & =-\frac{1}{2}\left|F_{21}\right|^{2}+(\text { a bounded function }), \\
x_{1}+\mathrm{i} x_{2} & =F_{11} \overline{F_{21}}-F_{12} \overline{F_{22}}=c \overline{F_{21}}+(\text { a bounded function }),
\end{aligned}
$$

where $c \in C \backslash\{0\}$ is a constant, we have $\Pi \circ f \rightarrow(0,0,-1)$ as $z \rightarrow 0$.

Next we assume the end is parabolic. Again we may set $G=z^{m}, m \in$ $Z_{+}$. Applying Proposition 2.1 for $t=2 m \pi$ and $\varepsilon=-1$, there exists a PSU PS1- $_{1,-}$ lift $g_{0}$ of $S(g)$ such that $h(z):=R \star g_{0}(z)+2 m \mathrm{i} \log z$ is a single-valued meromorphic function on $\Delta^{*}$, and the secondary Gauss map $g$ satisfies $g=A \star g_{0}$ or $1 / g=A \star g_{0}$ for some $A \in \mathrm{SU}_{1,1}$. By completeness, Lemma $\mathrm{P}$ implies that the end is of first kind. Hence by (2)(iii) of Proposition 2.1, $h(z)$ is holomorphic on a neighborhood of the origin. Moreover, by the assumption $|g|<1$, 
(2)(iv) of Proposition 2.1 yields that $g=A \star g_{0}$ for some $A \in \mathrm{SU}_{1,1}$. Thus, without loss of generality, we may set

$$
R \star g(z)=2 m \mathrm{i}(k(z)-\log z),
$$

here we set $h(z)=2 m \mathrm{i} k(z)$.

For a holomorphic null lift $F$ of $f$ with the secondary Gauss map $g$, set

$$
\hat{F}=F B^{-1}, \quad \text { where } \quad B:=\left(\left(\begin{array}{cc}
1 & 0 \\
0 & 2 \mathrm{i}
\end{array}\right) R\right)=\left(\begin{array}{cc}
1 / 2 & 1 / 2 \\
-1 & 1
\end{array}\right) \in \mathrm{SL}_{2} \boldsymbol{C} .
$$

Then $\hat{F}$ is a holomorphic null immersion whose hyperbolic Gauss map $\hat{G}$ and secondary Gauss map $\hat{g}$ are given by

$$
\begin{aligned}
\hat{G} & =G=z^{m}, \\
\hat{g}(z) & =B \star g(z)=\left(\begin{array}{cc}
1 & 0 \\
0 & 2 \mathrm{i}
\end{array}\right) \star(R \star g(z))=m(k(z)-\log z) .
\end{aligned}
$$

So applying (1.10) for this $(\hat{G}, \hat{g})$, the components of $\hat{F}$ are written as

$$
\begin{array}{ll}
\hat{F}_{11}(z)=-\frac{\mathrm{i}}{2} z^{m / 2} \varphi_{1}(z), & \hat{F}_{12}(z)=-\frac{\mathrm{i}}{2} z^{m / 2}\left(m \varphi_{1}(z) \log z+\psi_{1}(z)\right), \\
\hat{F}_{21}(z)=\frac{\mathrm{i}}{2} z^{-m / 2} \varphi_{2}(z), & \hat{F}_{22}(z)=\frac{\mathrm{i}}{2} z^{-m / 2}\left(m \varphi_{2}(z) \log z+\psi_{2}(z)\right),
\end{array}
$$

where $\varphi_{1}, \varphi_{2}, \psi_{1}$ and $\psi_{2}$ are holomorphic functions defined on a neighborhood of the origin such that

$$
\varphi_{1}(0)=\varphi_{2}(0)=1
$$

Since $B e_{3} B^{*}=-e_{1}, f=F e_{3} F^{*}$ satisfies

$$
f=-\hat{F}\left(\begin{array}{ll}
0 & 1 \\
1 & 0
\end{array}\right) \hat{F}^{*}=-\left(\begin{array}{ll}
\hat{F}_{11} \overline{\hat{F}_{12}}+\hat{F}_{12} \overline{\hat{F}_{11}} & \hat{F}_{11} \overline{\hat{F}_{22}}+\hat{F}_{12} \overline{\hat{F}_{21}} \\
\hat{F}_{11} \hat{F}_{22}+\overline{\hat{F}_{12}} \hat{F}_{21} & \hat{F}_{21} \overline{\hat{F}_{22}}+\hat{F}_{22} \overline{\hat{F}_{21}}
\end{array}\right) .
$$

Hence the components of $f$ are expressed as

$$
\begin{aligned}
x_{0} & =\frac{m}{4} r^{-m}\left(\eta_{1}(u, v) \log r+\delta_{1}(u, v)\right), \\
x_{3} & =\frac{m}{4} r^{-m}\left(-\eta_{2}(u, v) \log r+\delta_{2}(u, v)\right), \\
x_{1}+\mathrm{i} x_{2} & =-\frac{m}{2} \mathrm{e}^{\mathrm{i} m \theta}\left(\eta_{3}(u, v) \log r+\delta_{3}(u, v)\right),
\end{aligned}
$$


where $z=r \mathrm{e}^{\mathrm{i} \theta}=u+\mathrm{i} v$. Here, $\eta_{j}(u, v)(j=1,2)$ and $\delta_{j}(u, v)(j=1,2)$ (resp. $\eta_{3}(u, v)$ and $\left.\delta_{3}(u, v)\right)$ are real-valued (resp. complex-valued) differentiable functions defined on a neighborhood of the origin, such that $\eta_{j}(0,0)=1$ $(j=1,2,3)$.

Equations (4.7) yield that $x_{0} \rightarrow+\infty$ and $\Pi \circ f \rightarrow(0,0,-1)$ as $z \rightarrow 0$.

\section{The Osserman-type inequality}

Here we prove Theorem 0.2 stated in the introduction. First we prepare:

Lemma 5.1. The Hopf differential of a CMC-1 face has a pole of order 2 at any complete regular parabolic end.

Proof. Let $f: \Delta^{*} \rightarrow S_{1}^{3}$ be a complete regular parabolic end at $z=0$. By Theorem 3.1, the end is of the first kind. Then

$$
2 Q+S_{z}(G) d z^{2}=S_{z}(g) d z^{2}=\frac{1}{z^{2}}\left(\frac{1}{2}+o(1)\right) d z^{2} .
$$

Since $G$ is meromorphic at $z=0$, we may assume that $G=z^{m} \varphi(z)$, where $m$ is a positive integer and $\varphi(z)$ is a holomorphic function on a neighborhood of 0 with $\varphi(0) \neq 0$. Applying (1.19) to $S_{z}(G)$, it follows that $Q$ has a pole of order 2 at $z=0$.

The next lemma improves a result in [7, Proposition 4.4]:

Lemma 5.2. Let $f: \Delta^{*} \rightarrow S_{1}^{3}$ be a complete regular end at $z=0$ of a $C M C-1$ face with Hopf differential $Q$ and hyperbolic Gauss map G. Then the ramification order $m$ of $G(z)$ at $z=0$ satisfies

$$
m \geq \underset{z=0}{\operatorname{Ord}}(Q)+3 .
$$

(For the definition of the ramification order, see the subsection about the Schwarzian derivative in Section 1.) Here $\operatorname{Ord}_{z=0} Q$ denotes the order of $Q$ at the origin, that is, $\operatorname{Ord}_{z=0} Q=k$ if $Q=z^{k} \varphi(z) d z^{2}$, where $\varphi(z)$ is holomorphic at $z=0$ and $\varphi(0) \neq 0$.

Proof. By Theorem 3.1, a complete end is either elliptic or parabolic of the first kind. The elliptic case has been proved in [7]. Assume that the end is parabolic. Then by Lemma 5.1, $Q$ must have a pole of order 2 at $z=0$, which proves the inequality since $m \geq 1$. 
It should be remarked that the order of the metric $d \sigma_{\#}^{2}=4|d G|^{2} /\left(1+|G|^{2}\right)^{2}$ at 0 is equal to $m-1$, where $m$ is the ramification order of $G$. Using Lemma 5.2 instead of [7, Proposition 4.4], the inequality in Theorem 0.2 is proved in the same way as $[7,23]$.

The condition for equality in $(*)$ in Theorem 0.2 for elliptic ends was completely analyzed in [7]. So, it suffices to show the following theorem for parabolic ends. Note that $\operatorname{Ord}_{p}(Q)=-2$ for complete regular parabolic ends, hence the equality in (5.1) holds if and only if $G$ does not branch at $p$ (see [7] for details).

Theorem 5.1. A complete regular parabolic end of a CMC-1 face is properly embedded if and only if the hyperbolic Gauss map $G$ does not branch at the end.

Proof. Let $f: \Delta^{*} \rightarrow S_{1}^{3}$ be a complete regular parabolic end at $z=0$. Taking $-f$ instead of $f$ if necessary, we may assume that $|g|<1$ in a neighborhood of the end, and that $G(z)=z^{m}, m \geq 1$ and $g(z)=R^{-1} \star(2 m \mathrm{i}(k(z)-\log z))$, as in the proof of Proposition 4.2. Then $f$ is represented as in (4.5) and (4.6).

By Proposition 4.2, the image of $f$ tends to a point in $\partial_{+} S_{1}^{3}$. So we may assume that $x_{0}>1$ on $\Delta^{*}$, and

$$
\Pi \circ f: \Delta^{*} \ni z \longmapsto\left(X_{1}, X_{2}, X_{3}\right) \in \boldsymbol{R}^{3}
$$

is well defined, where $\Pi$ is the projection in (4.3).

Here, by (4.7),

$$
U(z):=z^{-m} \frac{x_{1}+\mathrm{i} x_{2}}{1+x_{0}}\left(=z^{-m}\left(X_{1}+\mathrm{i} X_{2}\right)\right)=-2 \frac{\eta_{3} \log r+\delta_{3}}{\eta_{1} \log r+\delta_{1}+(4 / m) r^{m}} .
$$

Since $\eta_{1}, \eta_{3}$ and $\delta_{1}, \delta_{3}$ are differentiable functions defined on a neighborhood of 0 , we have

(5.2) $\lim _{z \rightarrow 0} U(z)=-2 \neq 0, \quad \lim _{z \rightarrow 0} z \frac{\partial}{\partial z} U(z)=0 \quad$ and $\quad \lim _{z \rightarrow 0} z \frac{\partial}{\partial \bar{z}} U(z)=0$.

Now we suppose that the ramification order $m$ of the hyperbolic Gauss map at $z=0$ is 1 , that is $m=1$. As seen in the proof of Proposition 4.2, $\Pi \circ f$ converges to $(0,0,-1)$. Then $X_{1}+\mathrm{i} X_{2}=z U(z)$ and (5.2) yield that

$$
\lim _{z \rightarrow 0} \frac{\partial}{\partial z}\left(X_{1}+\mathrm{i} X_{2}\right) \neq 0 \quad \text { and } \quad \lim _{z \rightarrow 0} \frac{\partial}{\partial \bar{z}}\left(X_{1}+\mathrm{i} X_{2}\right)=0,
$$


which implies that the correspondence $z \mapsto X_{1}+\mathrm{i} X_{2}$ is bijective near the origin, and the end is properly embedded.

Conversely, suppose that the end is properly embedded. We have already seen that $X_{3} \rightarrow-1$ as $z \rightarrow 0$. Moreover $U(0) \neq 0$ implies that for any sufficiently small $\varepsilon>0$, the image of the end $f(\{z ; 0<|z|<\varepsilon\})$ does not meet the $X_{3}$-axis and is diffeomorphic to a cylinder. Then the image of $\Pi$ o $f(\{z ;|z|=\varepsilon\})$ by the orthogonal projection $\left(X_{1}, X_{2}, X_{3}\right) \mapsto X_{1}+\mathrm{i} X_{2}$ is an embedded closed curve with the winding number $m$ with respect to the origin. So $m=1$.

Remark 5.1. In Proposition 4.4 of [7], the first author showed the equality condition in Theorem 0.2 for elliptic ends using the expression of the solution of the ordinary differential equation (1.6). Here we proved the equality condition in Theorem 0.2 for parabolic ends by using Small's formula (1.10). It is also possible to prove the result in [7] more directly by using (1.10).

We give here three important examples:

Example 5.1 (An incomplete 3-noid not satisfying (*) in Theorem 0.2). We set $M^{2}:=\boldsymbol{C} \backslash\{0,1\}$ and

$$
G:=z, \quad g:=\frac{2 z-1}{2 z(z-1)}-\log \frac{z}{z-1} .
$$

Then (1.10) gives a CMC-1 face $f: M^{2} \rightarrow S_{1}^{3}$ with hyperbolic and secondary Gauss maps $G$ and $g$, and Hopf differential

$$
Q=\frac{1}{2}(S(g)-S(G))=-\frac{2 d z^{2}}{z(z-1)} .
$$

Since the lift metric

$$
d s_{\#}^{2}=\frac{4\left(1+|z|^{2}\right)^{2}}{|z(z-1)|^{2}}|d z|^{2}
$$

is complete on $M^{2}, f$ is weakly complete. The end $z=\infty$ is complete and elliptic, and $z=0,1$ are parabolic ends of the second kind. Hence $z=0,1$ are incomplete ends. Since $\operatorname{deg}(G)=1, f$ does not satisfy $(*)$. This implies that completeness is an essential assumption in Theorem 0.2 in the introduction. 
Example 5.2 (A 2-noid with complete parabolic ends satisfying the equality in $(*))$. We set

$$
F(z)=\frac{\mathrm{i}}{2 \sqrt{2}}\left(\begin{array}{cc}
\sqrt{z} & 0 \\
0 & \sqrt{z}^{-1}
\end{array}\right)\left(\begin{array}{cc}
3-\log z & -1+\log z \\
1+\log z & -3-\log z
\end{array}\right) .
$$

Then $f=F e_{3} F^{*}: \boldsymbol{C} \backslash\{0\} \rightarrow S_{1}^{3}$ has two parabolic regular ends. The hyperbolic Gauss map $G$, the secondary Gauss map $g$ and the Hopf differential $Q$ are computed as follows:

$$
G=z, \quad g=\frac{\log z+1}{\log z-1}, \quad Q=\frac{d z^{2}}{4 z^{2}} .
$$

Since $\{z \in \boldsymbol{C} ;|g(z)|=1\}=\{z \in \boldsymbol{C} ;|z|=1\}$, the singular set is compact, and hence $f$ is complete.

Any genus zero CMC-1 face with two parabolic regular ends and with degree 1 hyperbolic Gauss map is congruent to this $f$. We call this CMC-1 face the parabolic catenoid. On the other hand, the CMC-1 face with $G=z$, $g=z^{\mu}(\mu \in \boldsymbol{R} \backslash\{0\})$ given in [7, Example 5.4] is called the elliptic catenoid.

Example 5.3 (A complete 4-noid with 4 integral elliptic ends satisfying the equality in $(*))$. Since $\mathrm{SL}_{2} \boldsymbol{C}$ can be identified with the complex hyperquadric $Q^{3}$ of $\boldsymbol{C}^{4}$, the null (meromorphic) curves in $\mathrm{SL}_{2} \boldsymbol{C}$ can be identified with those in $Q^{3}$. The null curve in $\mathrm{SL}_{2} C$ with

$$
G:=\frac{3\left(z^{3}+2\right)}{4-z}, \quad g:=-\frac{z^{3}-12 z^{2}+2}{3 z}
$$

belongs to the moduli space $\mathscr{M}_{4}$ in the classification list of null curves in $Q^{3}$ in Bryant [4], which has four integral elliptic ends at the roots of $1+6 z^{2}-z^{3}$ and $z=\infty$. Since $G$ is of degree 3 and $\chi(C \cup\{\infty\})=2$, the corresponding CMC-1 face attains equality in $(*)$ of Theorem 0.2 . (For the definition of an integral elliptic end, see Definition 3.3.)

Remark 5.2. We can deform an elliptic catenoid to a parabolic catenoid. Let $f_{\mu}$ be an elliptic catenoid with the hyperbolic Gauss map $G=z$ and the secondary Gauss map $g=z^{\mu}$, where $\mu>0$. Then the hyperbolic metric corresponding to $f_{\mu}$ is

$$
d \sigma_{\mu}^{2}=\frac{4|d g|^{2}}{\left(1-|g|^{2}\right)^{2}}=\frac{4 \mu^{2}|z|^{2 \mu-2}}{\left(1-|z|^{2 \mu}\right)^{2}}|d z|^{2}
$$


It can be easily checked that

$$
\lim _{\mu \rightarrow 0} d \sigma_{\mu}^{2}=\frac{|d z|^{2}}{(r \log r)^{2}}, \quad \text { where } z=r \mathrm{e}^{\mathrm{i} \theta},
$$

which is the hyperbolic metric of a parabolic catenoid with

$$
g(z)=R \star \log z=\frac{1}{\mathrm{i}} \frac{\log z+1}{\log z-1},
$$

see (3.3). On the other hand, by Small's formula (1.10) there exists a unique smooth 1-parameter family of CMC-1 faces $\tilde{f}_{\mu}(\mu \geq 0)$ with hyperbolic Gauss map $G=z$ and associated hyperbolic metric $d \sigma_{\mu}^{2}$. Then $\tilde{f}_{\mu}$ is congruent to $f_{\mu}$, and $\tilde{f}_{0}$ gives a parabolic catenoid.

Remark 5.3. As a consequence of Remark 1.15, we know that there are no compact CMC-1 immersed surfaces in $S_{1}^{3}$. Here we give an alternative proof of this: Let $M^{2}$ be a compact Riemann surface without boundary, and suppose there exists a compact CMC-1 face $f: M^{2} \rightarrow S_{1}^{3}$ which has no singular points. Let $F$ be a holomorphic lift of $f$. We may assume that $|g|<1$ since there are no singular points. Then, by (1.6), we have

$$
f_{z \bar{z}}=\left(1-|g|^{2}\right) F\left(\begin{array}{l}
g \\
1
\end{array}\right)\left(\begin{array}{ll}
\bar{g} & 1
\end{array}\right) F^{*}|\hat{\omega}|^{2},
$$

where $z$ is a local complex coordinate and $\omega=\hat{\omega} d z$. Thus, trace $f$ is a nonconstant subharmonic function, which is a contradiction to the maximum principle.

This proof does not apply to compact CMC-1 faces, leading us to the following open problem:

Problem. Is there a compact CMC-1 face?

If such a CMC-1 face exists, the genus $\gamma$ must be greater than or equal to 3 , since equality in $(*)$ in the introduction holds in this case and the degree of the hyperbolic Gauss map must be $\gamma-1$.

\section{Appendix A. Meromorphicity of the Hopf differential}

In this appendix, we shall give a proof of the following 
Theorem A.1. Let $\bar{M}^{2}$ be a compact Riemann surface. Then the Hopf differential $Q$ of a complete $C M C-1$ face

$$
f: \bar{M}^{2} \backslash\left\{p_{1}, \ldots, p_{n}\right\} \longrightarrow S_{1}^{3}
$$

is meromorphic on $\bar{M}^{2}$.

Proof. It is sufficient to show the meromorphicity of $Q$ at a complete end $f: \Delta^{*}=\{z ; 0<|z|<1\} \rightarrow S_{1}^{3}$ at the origin. We write the Hopf differential $Q$ as

$$
Q=\hat{Q} d z^{2}
$$

where $\hat{Q}$ is a holomorphic function on $\Delta^{*}$. By Theorem 3.1, a complete end $f: \Delta^{*} \rightarrow S_{1}^{3}$ is either a $g$-regular elliptic end or a $g$-regular parabolic end of the first kind. (The definition of $g$-regularity is given in Definition 3.2.)

First, we consider the case that $f$ is elliptic. By $g$-regularity, the secondary Gauss map is written in the form

$$
g=z^{\mu} h(z) \quad(h \text { is a holomorphic function with } h(0) \neq 0),
$$

where $\mu$ is a real number. Since $|g(0)| \neq 1$ by completeness, we may set $g(0)=0$, or $\infty$, because of the $\mathrm{SU}_{1,1}$-ambiguity of $g$. Moreover, replacing $f$ by $-f$ if necessary, we may assume $\mu>0$ without loss of generality. In this case, the corresponding hyperbolic metric $d \sigma^{2}$ is written as

$$
d \sigma^{2}=\left(\frac{2|z|^{\mu-1}\left|\mu h(z)+z h^{\prime}(z)\right|}{\left.|1-| z\right|^{2 \mu}|h(z)|^{2} \mid}|d z|\right)^{2}, \quad\left({ }^{\prime}=\frac{d}{d z}\right)
$$

Since $|z|^{\mu}|h(z)|$ and $z h^{\prime}(z)$ tend to 0 as $z \rightarrow 0$ and $h(z)$ is bounded near the origin, we have that

$$
d \sigma \geq c|z|^{\mu-1}|d z| \geq c|z|^{l}|d z|
$$

holds on a neighborhood of the origin, where $l$ is the smallest integer such that $l \geq \mu-1$ and $c$ is a positive constant. Then, by (1.16), we have

$$
d s=2 \frac{|Q|}{d \sigma} \leq 2 \frac{|\hat{Q}|}{c|z|^{l}}|d z|=\frac{2}{c}\left|\frac{\hat{Q}}{z^{l}} d z\right|
$$

Since $d s$ is complete at 0 , we have meromorphicity of the one-form $z^{-l} \hat{Q} d z$ at the origin, because of [16, Lemma 9.6, page 83]. 
Next, we consider the case that $f$ is parabolic. Since the end is $g$-regular parabolic of the first kind, one can choose the secondary Gauss map $g$ as in (3.2):

$$
g=R^{-1} \hat{g}, \quad \hat{g}(z)=\mathrm{i}(h(z) \pm \log z),
$$

where $h(z)$ is a holomorphic function on $\Delta:=\Delta^{*} \cup\{0\}$. Hence $d \sigma^{2}$ is written as in (3.3):

$$
d \sigma^{2}=\left(\frac{\left|h^{\prime}(z) \pm(1 / z)\right|}{|\operatorname{Re} h(z) \pm \log | z||}|d z|\right)^{2} \quad\left({ }^{\prime}=\frac{d}{d z}\right) .
$$

Since $z \log |z| \rightarrow 0$ as $z \rightarrow 0$ and $h$ is bounded on a neighborhood of the origin,

$$
d \sigma=\frac{\left|1 \pm z h^{\prime}(z)\right|}{|z \log | z||\left|1 \pm \frac{\operatorname{Re} h(z)}{\log |z|}\right|}|d z| \geq c|d z|
$$

holds on a neighborhood of the origin, where $c$ is a positive constant. Thus,

$$
d s=2 \frac{|Q|}{d \sigma} \leq \frac{2}{c}|\hat{Q} d z| .
$$

Hence, by the same argument as in the elliptic case, we have meromorphicity of $Q$ at the origin.

\section{Appendix B. Conjugacy classes of $\mathrm{SU}_{1,1}$}

The Lie group $\mathrm{SU}_{1,1}$ is the set of matrices $S \in \mathrm{SL}_{2} C$ satisfying $S e_{3} S^{*}=e_{3}$. Two matrices $A, B \in \mathrm{SU}_{1,1}$ are called conjugate in $\mathrm{SL}_{2} C$ if there exists a matrix $P \in \mathrm{SL}_{2} C$ such that $B=P^{-1} A P$, and are called conjugate in $\mathrm{SU}_{1,1}$ if $B=P^{-1} A P$ for some $P \in \mathrm{SU}_{1,1}$.

As in (1.18) and (2.2), we set

$$
\begin{aligned}
& \Lambda_{\mathrm{e}}(t):=\left(\begin{array}{cc}
\mathrm{e}^{\mathrm{i} t} & 0 \\
0 & \mathrm{e}^{-\mathrm{i} t}
\end{array}\right), \\
& \Lambda_{\mathrm{p}}(t):=\left(\begin{array}{cc}
1+\mathrm{i} t & -\mathrm{i} t \\
\mathrm{i} t & 1-\mathrm{i} t
\end{array}\right), \\
& \Lambda_{\mathrm{h}}(t):=\left(\begin{array}{ll}
\cosh t & \sinh t \\
\sinh t & \cosh t
\end{array}\right) \quad \text { and } \quad R:=\frac{1}{2}\left(\begin{array}{rr}
1 & 1 \\
\mathrm{i} & -\mathrm{i}
\end{array}\right)
\end{aligned}
$$

for an arbitrary $t \in \boldsymbol{R}$. 
Theorem B.1. A matrix $A \in \mathrm{SU}_{1,1}$ is conjugate in $\mathrm{SU}_{1,1}$ to one of

1) $\Lambda_{\mathrm{e}}(s)(s \in(-\pi, \pi])$,

2) $\pm \Lambda_{\mathrm{p}}(t)$ or $\pm \Lambda_{\mathrm{p}}(-t)(t>0)$, or

3) $\pm \Lambda_{\mathrm{h}}(t)(t>0)$.

Remark B.1. Though the matrices $\Lambda_{\mathrm{e}}(s)$ and $\Lambda_{\mathrm{e}}(-s)$ are conjugate in $\mathrm{SL}_{2} \boldsymbol{C}$, they are not conjugate in $\mathrm{SU}_{1,1}$ if $s \not \equiv 0(\bmod 2 \pi)$. That is, for any elliptic matrix $A \in \mathrm{SU}_{1,1}$, there exists a unique real number $t \in(-\pi, \pi]$ such that $A$ and $\Lambda_{\mathrm{e}}(s)$ are conjugate in $\mathrm{SU}_{1,1}$.

Remark B.2. On the other hand, $\Lambda_{\mathrm{p}}\left(t_{1}\right)$ and $\Lambda_{\mathrm{p}}\left(t_{2}\right)\left(t_{1}, t_{2} \neq 0\right)$ are conjugate in $\mathrm{SU}_{1,1}$ if and only if $t_{1} t_{2}>0$. In fact, if $t_{1} \neq t_{2}, P \Lambda_{\mathrm{p}}\left(t_{1}\right) P^{-1}=\Lambda_{\mathrm{p}}\left(t_{2}\right)$ $\left(P \in \mathrm{SU}_{1,1}\right)$ holds if and only if

$$
P= \pm\left(\begin{array}{ll}
a & \bar{b} \\
b & \bar{a}
\end{array}\right), \quad a=\cosh s+\mathrm{i} u, \quad b=\sinh s+\mathrm{i} u,
$$

where $s=\log \sqrt{t_{2} / t_{1}} \in \boldsymbol{R}$ and $u \in \boldsymbol{R}$.

In particular, the sign of $t$ in $\Lambda_{\mathrm{p}}(t)$ is invariant under such a conjugation. Though $\Lambda_{\mathrm{p}}(t)(t \in \boldsymbol{R} \backslash\{0\})$ is conjugate with $\Lambda_{\mathrm{p}}(1)$ or $\Lambda_{\mathrm{p}}(-1)$, we choose various values of $t$ in this paper for the sake of convenience.

Remark B.3. Since

$$
\left(\begin{array}{rr}
\mathrm{i} & 0 \\
0 & -\mathrm{i}
\end{array}\right)\left(\begin{array}{ll}
\cosh t & \sinh t \\
\sinh t & \cosh t
\end{array}\right)\left(\begin{array}{rr}
-\mathrm{i} & 0 \\
0 & \mathrm{i}
\end{array}\right)=\left(\begin{array}{cc}
\cosh t & -\sinh t \\
-\sinh t & \cosh t
\end{array}\right)
$$

$\Lambda_{\mathrm{h}}(t)$ and $\Lambda_{\mathrm{h}}(-t)$ are conjugate in $\mathrm{SU}_{1,1}$.

To show Theorem B.1, we use the following group isomorphism:

$$
\rho: \mathrm{SL}_{2} \boldsymbol{R} \ni X \longmapsto R^{-1} X R \in \mathrm{SU}_{1,1} \text {. }
$$

Note that $\Lambda_{\mathrm{e}}(t), \Lambda_{\mathrm{p}}(t)$ and $\Lambda_{\mathrm{h}}(t)$ are the images of

$$
\left(\begin{array}{cc}
\cos t & \sin t \\
-\sin t & \cos t
\end{array}\right), \quad\left(\begin{array}{cc}
1 & 2 t \\
0 & 1
\end{array}\right) \text { and }\left(\begin{array}{cc}
\mathrm{e}^{t} & 0 \\
0 & \mathrm{e}^{-t}
\end{array}\right)
$$

respectively, by $\rho$.

Lemma B.1. Let $A$ and $B$ be $2 \times 2$ real matrices that are conjugate in $\mathrm{SL}_{2} C$. Then $A$ and either $B$ or $e_{3} B e_{3}$ are conjugate in $\mathrm{SL}_{2} \boldsymbol{R}$. 
Proof. By assumption, there is a $\widetilde{P} \in \mathrm{SL}_{2} C$ with $A \widetilde{P}=\widetilde{P} B$. We set $\widetilde{P}=$ $U+\mathrm{i} V$, for real matrices $U, V$. Then $A U=U B, A V=V B$ and

$$
A(U+t V)=(U+t V) B \text { for any } t \in \boldsymbol{R} .
$$

If $\operatorname{det}(U+t V)$ vanishes identically for all $t \in \boldsymbol{R}$, holomorphicity of $\boldsymbol{C} \ni t \mapsto$ $\operatorname{det}(U+t V)$ yields that $\operatorname{det}(U+\mathrm{i} V)=\operatorname{det} \widetilde{P}=0$, a contradiction. Thus for some $t_{0} \in \boldsymbol{R}, \operatorname{det}\left(U+t_{0} V\right) \neq 0$, and then $\left(U+t_{0} V\right)^{-1} A\left(U+t_{0} V\right)=B$. If $\operatorname{det}\left(U+t_{0} V\right)>0$, we set $P=\left(U+t_{0} V\right) / \sqrt{\operatorname{det}\left(U+t_{0} V\right)} \in \mathrm{SL}_{2} \boldsymbol{R}$, giving $P^{-1} A P=B$. If $\operatorname{det}\left(U+t_{0} V\right)<0$, we set

$$
P=\frac{\left(U+t_{0} V\right) e_{3}}{\sqrt{\left|\operatorname{det}\left(U+t_{0} V\right)\right|}} \in \mathrm{SL}_{2} \boldsymbol{R}
$$

giving $P^{-1} A P=e_{3} B e_{3}$.

Proof of Theorem B.1. Let $A \in \mathrm{SU}_{1,1}$ and $\widetilde{A}:=\rho^{-1}(A) \in \mathrm{SL}_{2} \boldsymbol{R}$.

If the eigenvalues of $A$ are not real numbers, they are written as $\left\{\mathrm{e}^{\mathrm{i} t}, \mathrm{e}^{-\mathrm{i} t}\right\}$, where $t \in(-\pi, 0) \cup(0, \pi)$. In this case, $\widetilde{A}$ is conjugate in $\mathrm{SL}_{2} \boldsymbol{C}$ to $B_{\mathrm{e}}:=R \Lambda_{\mathrm{e}}(t) R^{-1}$. Hence by Lemma B.1, $\widetilde{A}$ is conjugate in $\mathrm{SL}_{2} \boldsymbol{R}$ to $B_{\mathrm{e}}$ or $e_{3} B_{\mathrm{e}} e_{3}$. Thus, $A=\rho(\widetilde{A})$ is conjugate in $\mathrm{SU}_{1,1}$ to $\rho\left(B_{\mathrm{e}}\right)=\Lambda_{\mathrm{e}}(t)$ or $\rho\left(e_{3} B_{\mathrm{e}} e_{3}\right)=\Lambda_{\mathrm{e}}(-t)$.

If the eigenvalues of $A$ are $\{\varepsilon, \varepsilon\}(\varepsilon=\{-1,1\})$ and $A \neq \varepsilon$ id, $\widetilde{A}$ is conjugate in $\mathrm{SL}_{2} C$ to $B_{\mathrm{p}}:=\varepsilon R \Lambda_{\mathrm{p}}(t) R^{-1}$ for any $t \in \boldsymbol{R}_{+}$. Hence $\widetilde{A}$ is conjugate in $\mathrm{SL}_{2} \boldsymbol{R}$ to either $B_{\mathrm{p}}$ or $e_{3} B_{\mathrm{p}} e_{3}$. Thus, $A$ is conjugate in $\mathrm{SU}_{1,1}$ to $\rho\left(B_{\mathrm{p}}\right)=\varepsilon \Lambda_{\mathrm{p}}(t)$ or $\rho\left(e_{3} B_{\mathrm{p}} e_{3}\right)=\varepsilon \Lambda_{\mathrm{p}}(-t)$. As mentioned in Remark B.2, $\Lambda_{\mathrm{p}}(u)$ for $u \in \boldsymbol{R} \backslash\{0\}$ is conjugate in $\mathrm{SU}_{1,1}$ to $\Lambda_{\mathrm{p}}(\operatorname{sgn} u)=\Lambda_{\mathrm{p}}(\varepsilon)$.

If the eigenvalues of $A$ are two distinct real numbers, they are represented as $\left\{\varepsilon \mathrm{e}^{t}, \varepsilon \mathrm{e}^{-t}\right\}$, where $t \in \boldsymbol{R}_{+}$and $\varepsilon \in\{-1,1\}$. Thus, $\widetilde{A}$ is conjugate in $\mathrm{SL}_{2} \boldsymbol{C}$ to the diagonal matrix $B_{\mathrm{h}}:=\varepsilon R \Lambda_{\mathrm{h}}(t) R^{-1}$. Hence, similarly to the first case, $A$ is conjugate in $\mathrm{SU}_{1,1}$ to $\rho\left(B_{\mathrm{h}}\right)=\varepsilon \Lambda_{\mathrm{h}}(t)$.

\section{References}

[1] R. Aiyama and K. Akutagawa, Kenmotsu-Bryant type representation formulas for constant mean curvature surfaces in $H^{3}\left(-c^{2}\right)$ and $S_{1}^{3}\left(c^{2}\right)$, Ann. Global Anal. Geom. 17 (1998), 49-75. 
[2] K. Akutagawa, On spacelike hypersurfaces with constant mean curvature in the de Sitter space, Math. Z. 196 (1987), 13-19.

[3] R. Bryant, Surfaces of mean curvature one in hyperbolic space, Astérisque, 154-155 (1987), 321-347.

[4] R. Bryant, Surfaces in conformal geometry, Proc. Symp. Pure Math., 48 (1988), 227-240.

[5] E. A. Coddington and N. Levinson, Theory of ordinary differential equations, McGraw-Hill, New York, 1955.

[6] P. Collin, L. Hauswirth and H. Rosenberg, The geometry of finite topology Bryant surfaces, Ann. Math. 153 (2001), 623-659.

[7] S. Fujimori, Spacelike CMC 1 surfaces with elliptic ends in de Sitter 3-Space, Hokkaido Math. J. 35 (2006), 289-320.

[8] S. Fujimori, W. Rossman, M. Umehara, K. Yamada and S.-D. Yang, New maximal surfaces in Minkowski 3-space with arbitrary genus and their CMC-1 cousins in de Sitter 3-space, Preprint.

[9] S. Fujimori, K. Saji, M. Umehara and K. Yamada, Singularities of maximal surfaces, Math. Z. 259 (2008), 827-848.

[10] J. Gálvez, A. Martínez and F. Milán, Complete linear Weingarten surfaces of Bryant type. A Plateau problem at infinity, Trans. Amer. Math. Soc. 356 (2004), 3405-3428.

[11] O. Kobayashi, Maximal surfaces in the 3-dimensional Minkowski space $\mathbb{L}^{3}$, Tokyo J. Math. 6 (1983), 297-309.

[12] M. Kokubu and M. Umehara, Global properties of linear Weingarten surfaces of Bryant type in $H^{3}$, Preprint, arXiv:0907.2284.

[13] M. Kokubu, M. Umehara and K. Yamada, An elementary proof of Small's formula for null curves in $\mathrm{PSL}_{2} \boldsymbol{C}$ and an analogue for legendrian curves in $\mathrm{PSL}_{2} \boldsymbol{C}$, Osaka J. Math. 40 (2003), 697-715.

[14] M. Kokubu, M. Umehara and K. Yamada, Flat fronts in hyperbolic 3-space, Pacific J. Math. 216 (2004), 149-175.

[15] S. Lee and S.-D. Yang, Spacelike constant mean curvature 1 trinoids in de Sitter three-space, Osaka J. Math. 43 (2006), 641-663.

[16] R. Osserman, A survey of minimal surfaces, Dover Publications, New York, 1986. 
426 S. Fujimori, W. Rossman, M. Umehara, K. Yamada and S.-D. Yang

[17] J. Ramanathan, Complete spacelike hypersurfaces of constant mean curvature in de Sitter space, Indiana Univ. Math. J. 36 (1987), 349-359.

[18] W. Rossman, M. Umehara and K. Yamada, Mean curvature 1 surfaces in hyperbolic 3-space with low total curvature I, Hiroshima Math. J. 34 (2004), 21-56.

[19] A. J. Small, Surface of constant mean curvature in $H^{3}$ and algebraic curves on a quadric, Proc. Amer. Math. Soc. 122 (1994), 1211-1220.

[20] M. Troyanov, Metric of constant curvature on a sphere with two conical singularities, in "Differential Geometry" (F. J. Carreras, O. Gil-Medrano, A. M. Naveira (Eds.)) Lect. Notes in Math., 1410, (1989), pp. 296-308, Springer-Verlag.

[21] M. Umehara and K. Yamada, Complete surfaces of constant mean curvature one in the hyperbolic 3-space, Ann. Math. 137 (1993), 611-638.

[22] M. Umehara and K. Yamada, Surfaces of constant mean curvature c in $H^{3}\left(-c^{2}\right)$ with prescribed hyperbolic Gauss map, Math. Ann. 304 (1996), 203-224.

[23] M. Umehara and K. Yamada, A duality on CMC-1 surfaces in hyperbolic space, and a hyperbolic analogue of the Osserman inequality, Tsukuba J. Math. 21 (1997), 229-237.

[24] M. Umehara and K. Yamada, Maximal surfaces with singularities in Minkowski space, Hokkaido Math. J. 35 (2006), 13-40.

[25] M. Umehara and K. Yamada, Applications of a completeness lemma in minimal surface theory to various classes of surfaces, Preprint, arXiv:0909.1128.

[26] Z.-H. Yu, Value distribution of hyperbolic Gauss map, Proc. Amer. Math. Soc. 125 (1997), 2997-3001.

SHOICHI FujIMORI

Department of Mathematics

FUKUOKA UNIVERSITY OF EDUCATION

MUNAKATA

FUKUOKA 811-4192

JAPAN

E-mail address: fujimori@fukuoka-edu.ac.jp 
WAYNE ROSSMAN

Department of Mathematics

FACUlty of Science

KoBe UNIVERSITY

ROKKO

KoBe 657-8501

JAPAN

E-mail address: wayne@math.kobe-u.ac.jp

MASAAKI UMEHARA

Department of Mathematics

Graduate School of Science

OSAKA UNIVERSITY

TOYONAKA

OSAKA 560-0043

JAPAN

E-mail address: umehara@math.sci.osaka-u.ac.jp

Kotaro Yamada

Department of Mathematics

Tokyo Institute of TeChNology

O-OKayama, Meguro

TOKYO 152-8551

JAPAN

E-mail address: kotaro@math.titech.ac.jp

SEONG-DEOG YANG

Department of Mathematics

Korea University

SEOUL 136-701

KoreA

E-mail address: sdyang@korea.ac.kr

ReCEIVED June 06, 2007 
